IMA Journal of Numerical Analysis (2014) 34, 1361-1385

doi:10.1093/imanum/drt050

Advance Access publication on November 1, 2013

\title{
A decoupled and unconditionally convergent linear FEM integrator for the Landau-Lifshitz-Gilbert equation with magnetostriction
}

\author{
L'UBOMír BAŇAS \\ Faculty of Mathematics, Bielefeld University, Postfach 100131, D-33501 Bielefeld, Germany \\ Banas@math.uni-bielefeld.de \\ Marcus Page* and Dirk Praetorius \\ Institute for Analysis and Scientific Computing, Vienna University of Technology, Wiedner Hauptstraße \\ 8-10, A-1040 Wien, Austria \\ *Corresponding author: marcus.page@tuwien.ac.at dirk.praetorius@tuwien.ac.at \\ AND \\ JONATHAN ROCHAT \\ MATHICSE, École Polytechnique Fédérale de Lausanne, station 8, CH-1015 Lausanne, Switzerland \\ Jonathan.Rochat@epfl.ch
}

[Received on 20 March 2013; revised on 21 August 2013]

\begin{abstract}
To describe and simulate dynamic micromagnetic phenomena, we consider a coupled system of the nonlinear Landau-Lifshitz-Gilbert equation and the conservation of momentum equation. This coupling allows one to include magnetostrictive effects into the simulations. Existence of weak solutions has recently been shown in Carbou et al. (2011) (Global weak solutions for the Landau-Lifschitz equation with magnetostriction. Math. Meth. Appl. Sci., 34, 1274-1288). In our contribution, we give an alternate proof which additionally provides an effective numerical integrator. The latter is based on linear finite elements (FEs) in space and a linear-implicit Euler time-stepping. Despite the nonlinearity, only two linear systems have to be solved per timestep, and the integrator fully decouples both equations. Finally, we prove unconditional convergence — at least of a subsequence-towards, and hence existence of, a weak solution of the coupled system, as timestep size and spatial mesh size tend to zero. We conclude the work with numerical experiments, which study the discrete blow-up of the LLG equation as well as the influence of the magnetostrictive term on the discrete blow-up.
\end{abstract}

Keywords: LLG; magnetostriction; ferromagnetism; unconditionally convergent linear integrator.

\section{Introduction}

Throughout all technical areas, magnetic devices like sensors, recording heads and magneto-resistive storage devices are quite popular and thus widely used. As their size decreases to a microscale, and the testing and development becomes more and more involved, the need for reliable and stable simulation tools, as well as for a thorough theoretical understanding, rises. In terms of mathematical physics, micromagnetic phenomena are modelled best by the Landau-Lifshitz-Gilbert (LLG) equation, see (2.1). This nonlinear partial differential equation (PDE) describes the behaviour of the magnetization of some ferromagnetic body under the influence of a so-called effective field. The mathematical challenges, as well as its applicability to a wide range of real-world problems, make LLG an interesting problem not 
only for mathematicians and physicists, but also for scientists from related fields like engineers and developers from high-tech industry.

In our contribution, we present and analyse a computationally attractive integrator to solve LLG numerically. Additionally, our analysis provides a constructive existence proof for weak solutions of the coupled system for LLG with magnetostriction and thus particularly includes the results of Carbou et al. (2011). For pure LLG, existence and nonuniqueness of weak solutions goes back to Visintin (1985) and Alouges \& Soyeur (1992) for a simplified effective field. For a review of the analysis of LLG, we refer to Kružík \& Prohl (2006), García-Cervera (2007) and Cimrak (2008) or the monographs (Hubert \& Schäfer, 1998; Prohl, 2001) and the references therein. As far as the numerical analysis is concerned, mathematically reliable and convergent LLG integrators are found in Bartels \& Prohl (2006), Alouges (2008), Baňas et al. (2008), Bartels et al. (2008), Alouges et al. (2011), Goldenits et al. (2011), Bruckner et al. (2012), Goldenits et al. (2012), Le \& Tran (2012), Rochat (2012) and Baňas et al. (2013). Of particular interest are unconditionally convergent integrators, which do not impose a coupling of spatial mesh size $h$ and timestep size $k$ to ensure stability of the numerical scheme. Those integrators are split into two groups; first, midpoint-scheme-based integrators (Baňas et al., 2008; Rochat, 2012), which rely on the seminal work (Bartels \& Prohl, 2006); secondly, projection-based first-order integrators (Alouges et al., 2011; Goldenits et al., 2011; Bruckner et al., 2012; Goldenits et al., 2012; Le \& Tran, 2012; Baňas et al., 2013), which build on the seminal work (Alouges, 2008) and are the basis for our scheme. All of the above integrators have in common that they allow for constructive existence proofs of the corresponding problems. The idea, which is also exploited in the current work, is to show boundedness of the computable discrete solutions. Then, a compactness argument concludes the existence of weakly convergent subsequences. Finally, those weak limits are identified as weak solutions of the coupled system.

In our contribution, we extend the original idea of Alouges (2008) for projection-based integrators and show that its advantages can also be transferred to the coupled system of LLG with magnetostriction, where our algorithm decouples both problems. Even though the structure of the main proof in this work is similar to the one from, e.g., Alouges (2008), Baňas et al. (2013) and Bruckner et al. (2012) (and also the other works mentioned), the analysis is less straightforward as the individual ingredients are harder to gain than for the coupling with stationary equations (Bruckner et al., 2012) or with the nonstationary Maxwell system (Baňas et al., 2013). First, unlike the Maxwell-LLG system, the coupling to the conservation of momentum equation involves a nonlinear coupling operator, and this operator differs between both equations. Secondly, the field contribution which accounts for magnetostriction, does not only depend on the displacement, but also on its spatial derivative which therefore needs to be bounded as well. For these two reasons, the original analysis has to be extended at various points. Moreover, the proposed scheme fully decouples the two equations while still maintaining unconditional convergence. To that end, even the difference of two subsequent discrete solutions needs to be controlled. We also refer the reader to the discussion after Theorem 5.1. In contrast to the existing literature, we thus extend the approach from Alouges (2008) and analyse a nonlinear coupling of LLG to a second time-dependent PDE. The contributions of this work as well as the advances over the state of the art can be summarized as follows:

- We include the magnetostrictive field contribution into the numerical analysis as well as into the algorithm to account for elastic effects on a microscale. This allows one to conduct more precise simulations in certain applications.

- We give a new and constructive proof for the existence of weak solutions for LLG with magnetostriction by providing a numerical integrator, which is mathematically guaranteed to converge to a weak solution of the coupled system of LLG with magnetostriction. 
- As in the original analysis (Alouges, 2008), the proposed integrator is unconditionally convergent towards a weak solution as soon as timestep size $k$ and spatial mesh size $h$ tend to zero, independently of each other. While this is more or less a consequence of the scheme from Alouges (2008) and might be expected for straightforward discretizations, it is not obvious that the result carries over to a fully decoupled algorithm. For midpoint-scheme-based integrators (Bartels \& Prohl, 2006; Baňas et al., 2008; Rochat, 2012), unconditional convergence is theoretically proved. However, the solution of the nonlinear system requires either a heuristical solver or an appropriate fixedpoint iteration. The latter is used in Bartels \& Prohl (2006), Baňas et al. (2008) and Rochat (2012), but the resulting explicit scheme again involves a coupling of $h$ and $k$ to guarantee convergence and avoid instabilities.

- The proposed algorithm is extremely attractive from a computational point of view: Since the two equations are fully decoupled, the implementation is easy and only two subsequent linear systems have to be solved per timestep. Moreover, existing code as well as preconditioners can be reused. Contrary, midpoint-scheme-based integrators (Bartels \& Prohl, 2006; Baňas et al., 2008; Rochat, 2012) have to solve one large nonlinear system per timestep (see Baňas et al., 2008). The current work is thus the first one to present a rigorously analysed and unconditionally convergent decoupled scheme for the problem at hand.

- We provide a numerical comparison between the extended Alouges-type integrator and an integrator based on the midpoint scheme (Rochat, 2012). Empirically, the numerical results show that the proposed integrator works with larger timesteps than the midpoint scheme. In particular, in our setting the computations based on the Alouges-type integrator turn out to be much faster. This gives empirical evidence that the fixed-point iteration for the midpoint scheme is indeed a computational bottleneck.

Outline. The remainder of this paper is organized as follows: In Section 2, we state the mathematical model for the coupled system of LLG with magnetostriction and recall the notion of a weak solution (Definition 2.1). In Section 3, we collect some notation and preliminaries, as well as the definition of the discrete ansatz spaces. In Section 4, we write down our numerical integrator in Algorithm 4.1, and Section 5 is devoted to our main convergence result (Theorem 5.1) and its proof. Finally, numerical examples conclude the work in Section 6.

\section{Model problem}

The evolution of the magnetization of a ferromagnetic body $\Omega$ during some time interval $(0, T)$ is mathematically modelled by the LLG equation, which in dimensionless form reads

$$
\mathbf{m}_{t}-\alpha \mathbf{m} \times \mathbf{m}_{t}=-\mathbf{m} \times \mathbf{H}_{\mathrm{eff}} \quad \text { in } \Omega_{T}:=(0, T) \times \Omega .
$$

Here, $\mathbf{m}: \Omega_{T} \rightarrow \mathbb{S}^{2}:=\left\{\mathbf{x} \in \mathbb{R}^{3}:|\mathbf{x}|=1\right\}$ denotes the sought magnetization, and $\mathbf{H}_{\text {eff }}$ is the so-called effective field that consists of several energy contributions, each of which models a certain micromagnetic effect. More precisely, in our work, the effective field consists of the exchange contribution $\Delta \mathbf{m}$, the magnetostrictive component $\mathbf{h}_{\mathbf{m}}$ and all other stationary and lower-order effects are collected in some general field contribution $\pi(\mathbf{m})$, i.e.,

$$
\mathbf{H}_{\mathrm{eff}}=C_{e} \Delta \mathbf{m}+\mathbf{h}_{\mathbf{m}}-\boldsymbol{\pi}(\mathbf{m}) .
$$


The general energy contribution $\boldsymbol{\pi}(\mathbf{m})$ is only assumed to fulfil a certain set of properties, see (5.3-5.4), and we emphasize that this particularly includes the case $\mathbf{H}_{\mathrm{eff}}=C_{e} \Delta \mathbf{m}+\mathbf{h}_{\mathbf{m}}+C_{\mathrm{ani}} D \Phi(\mathbf{m})+P(\mathbf{m})-$ f. Here, $\Phi(\mathbf{m})$ denotes the crystalline anisotropy density and $\mathbf{f}$ is a given applied field. The contribution $P(\mathbf{m})$ stands for the nonlocal stray field. The constants $C_{e}$ and $C_{\text {ani }}>0$ denote the exchange and anisotropy constant, respectively. To keep the presentation simple, we did not include the additional coupling to the full Maxwell equations as in Baňas et al. (2013). We stress, however, that this extension is straightforward and, with the combined techniques from this work and Baňas et al. (2013), one could consider a coupled system of full Maxwell LLG with the conservation of momentum equation to account for magnetostrictive effects. The combined results from Baňas et al. (2013) and the current work would then directly transfer to the coupled case, and we would still derive unconditional convergence. Moreover, with the techniques from Bruckner et al. (2012), it is straightforward to rigorously include a numerical approximation $\pi_{h}(\cdot)$ of $\pi(\cdot)$ into the convergence analysis.

For a bounded Lipschitz domain $\Omega \subset \mathbb{R}^{3}$ and a time interval $(0, T)$, we now aim to solve (2.1a) on $\Omega_{T}$ supplemented by the initial and boundary conditions

$$
\mathbf{m}(0)=\mathbf{m}^{0} \in \mathbf{H}^{1}\left(\Omega ; \mathbb{S}^{2}\right) \quad \text { and } \quad \partial_{n} \mathbf{m}=0 \quad \text { on }(0, T) \times \partial \Omega .
$$

The constraint $\left|\mathbf{m}^{0}\right|=1$ almost everywhere in $\Omega$ models the fact that we consider a constant temperature below the Curie point. Multiplication of (2.1a) with $\mathbf{m}$ yields $\partial_{t}|\mathbf{m}|^{2}=2 \mathbf{m} \cdot \mathbf{m}_{t}=0$. Hence, the modulus $|\mathbf{m}|=1$ is preserved in time. By imposing the modulus constraint on $\mathbf{m}^{0}$, this guarantees $|\mathbf{m}(t)|=1$ for almost all times $t \in(0, T)$.

For modelling the magnetostrictive component, we follow the approach of Visintin (1985). Here, the magnetostrictive field reads

$$
\mathbf{h}_{\mathbf{m}}: \Omega_{T} \rightarrow \mathbb{R}^{3}, \quad\left(\mathbf{h}_{\mathbf{m}}\right)_{q}=\left(\mathbf{h}_{\mathbf{m}}(\mathbf{u}, \mathbf{m})\right)_{q}:=\sum_{i, j, p=1}^{3} \lambda_{i j p q}^{m} \sigma_{i j}(\mathbf{m})_{p},
$$

where $(\cdot)_{p}$ denotes the $p$ th component of a vector field. We implicitly assume linear dependence of the stress tensor $\sigma=\left\{\sigma_{i j}\right\}$ on the elastic part of the total strain $\boldsymbol{\varepsilon}^{e}=\left\{\varepsilon_{i j}^{e}\right\}$, which is the converse form of Hook's law, i.e.,

$$
\begin{aligned}
\sigma & :=\lambda^{e} \boldsymbol{\varepsilon}^{e}(\mathbf{u}, \mathbf{m}): \Omega_{T} \longrightarrow \mathbb{R}^{3 \times 3}, \quad \sigma_{i j}=\sum_{p, q=1}^{3} \lambda_{i j p q}^{e} \varepsilon_{p q}^{e}, \\
\boldsymbol{\varepsilon}^{e}(\mathbf{u}, \mathbf{m}) & :=\boldsymbol{\varepsilon}(\mathbf{u})-\boldsymbol{\varepsilon}^{m}(\mathbf{m}):[0, T] \times \Omega \longrightarrow \mathbb{R}^{3 \times 3},
\end{aligned}
$$

where $\mathbf{u}: \Omega_{T} \rightarrow \mathbb{R}^{3}$ denotes the displacement vector field. The total strain is defined by the symmetric part of the gradient of $\mathbf{u}$, i.e.,

$$
\varepsilon_{i j}(\mathbf{u}):=\frac{1}{2}\left(\frac{\partial u_{i}}{\partial x_{j}}+\frac{\partial u_{j}}{\partial x_{i}}\right),
$$

and the magnetic part of the total strain by

$$
\boldsymbol{\varepsilon}^{m}(\mathbf{m}):=\lambda^{m} \mathbf{m m}^{T}: \Omega_{T} \longrightarrow \mathbb{R}^{3 \times 3}, \quad \varepsilon_{i j}^{m}(\mathbf{m})=\sum_{p, q=1}^{3} \lambda_{i j p q}^{m}(\mathbf{m})_{p}(\mathbf{m})_{q} .
$$


In addition, we assume both material tensors $\lambda \in\left\{\lambda^{e}, \lambda^{m}\right\}$ to be symmetric $\left(\lambda_{i j p q}=\lambda_{j i p q}=\lambda_{i j q p}=\lambda_{p q i j}\right)$ and positive-definite

$$
\sum_{i, j, p, q=1}^{3} \lambda_{i j p q} \xi_{i j} \xi_{p q} \geqslant \lambda^{\star} \sum_{i, j=1}^{3} \xi_{i j}^{2}
$$

with bounded entries, i.e., there exists some $\bar{\lambda}$ with $\lambda_{i j p q}^{e}, \lambda_{i j p q}^{m} \leqslant \bar{\lambda}$ for any $i, j, p, q=1,2,3$. The stress tensor $\sigma$ and the displacement field $\mathbf{u}$ (where we assume no external forces) are finally coupled via the conservation of momentum equation

$$
\varrho \mathbf{u}_{t t}-\nabla \cdot \sigma=0 \quad \text { in } \Omega_{T} .
$$

Here, we assume the mass density $\varrho>0$ to be constant and independent of the deformation. Equation (2.8a) is additionally supplemented by the initial and boundary conditions

$$
\mathbf{u}(0)=\mathbf{u}^{0} \quad \text { in } \Omega, \quad \mathbf{u}_{t}(0)=\dot{\mathbf{u}}^{0} \quad \text { in } \Omega \quad \text { and } \quad \mathbf{u}=0 \quad \text { on } \partial \Omega .
$$

Altogether, we thus aim to solve the coupled problem

$$
\left\{\begin{array}{l}
\mathbf{m}_{t}-\alpha \mathbf{m} \times \mathbf{m}_{t}=-\mathbf{m} \times \mathbf{H}_{\mathrm{eff}}, \\
\varrho \mathbf{u}_{t t}-\nabla \cdot \boldsymbol{\sigma}=0
\end{array}\right.
$$

subject to the stated initial and boundary conditions.

Using the above boundary conditions, Hook's relation (2.3), the definition of the total strain tensor (2.4), and the symmetry of the tensors $\lambda^{e}$ and $\lambda^{m}$, we obtain the following variational formulation of (2.8a):

$$
\left(\varrho \mathbf{u}_{t t}(t), \boldsymbol{\varphi}\right)+\left(\lambda^{e} \boldsymbol{\varepsilon}(\mathbf{u})(t), \boldsymbol{\varepsilon}(\boldsymbol{\varphi})\right)=\left(\lambda^{e} \boldsymbol{\varepsilon}^{m}(\mathbf{m})(t), \boldsymbol{\varepsilon}(\boldsymbol{\varphi})\right) \quad \text { for all } \boldsymbol{\varphi} \in \mathbf{H}_{0}^{1}(\Omega) .
$$

Given these notations, we now define our notion of a weak solution for the coupled LLGmagnetostriction system (2.9), which is the same as in Carbou et al. (2011).

Definition 2.1 The tuple $(\mathbf{m}, \mathbf{u})$ is called a weak solution of LLG with magnetostriction, if for all $T>0$,

(i) $\mathbf{m} \in \mathbf{H}^{1}\left(\Omega_{T}\right)$ with $|\mathbf{m}|=1$ almost everywhere in $\Omega_{T}$ and $\mathbf{u} \in H^{1}\left(\Omega_{T}\right)$;

(ii) for all $\phi \in C^{\infty}\left(\Omega_{T}\right)$ and $\zeta \in C_{c}^{\infty}\left([0, T) ; C^{\infty}(\Omega)\right)$, we have

$$
\begin{aligned}
& \int_{\Omega_{T}}\left\langle\mathbf{m}_{t}, \boldsymbol{\phi}\right\rangle-\alpha \int_{\Omega_{T}}\left\langle\left(\mathbf{m} \times \mathbf{m}_{t}\right), \boldsymbol{\phi}\right\rangle \\
& \quad=-C_{\mathrm{exch}} \int_{\Omega_{T}}\langle(\nabla \mathbf{m} \times \mathbf{m}), \nabla \boldsymbol{\phi}\rangle+\int_{\Omega_{T}}\left\langle\left(\mathbf{h}_{\mathbf{m}} \times \mathbf{m}\right), \boldsymbol{\phi}\right\rangle-\int_{\Omega_{T}}\langle(\boldsymbol{\pi}(\mathbf{m}) \times \mathbf{m}), \boldsymbol{\phi}\rangle, \\
& -\varrho \int_{\Omega_{T}}\left\langle\mathbf{u}_{t}, \zeta_{t}\right\rangle+\int_{\Omega_{T}}\left\langle\lambda^{e} \boldsymbol{\varepsilon}(\mathbf{u}), \boldsymbol{\varepsilon}(\zeta)\right\rangle=\int_{\Omega_{T}}\left\langle\lambda^{e} \boldsymbol{\varepsilon}(\mathbf{m}), \boldsymbol{\varepsilon}(\zeta)\right\rangle+\int_{\Omega}\left\langle\dot{\mathbf{u}}^{0}, \zeta(0, \cdot)\right\rangle
\end{aligned}
$$

(iii) there holds $\mathbf{m}(0, \cdot)=\mathbf{m}^{0}$ and $\mathbf{u}(0, \cdot)=\mathbf{u}_{0}$ in the sense of traces; 
(iv) for almost all $t^{\prime} \in(0, T)$, we have bounded energy

$$
\left\|\nabla \mathbf{m}\left(t^{\prime}\right)\right\|_{\mathbf{L}^{2}(\Omega)}^{2}+\left\|\mathbf{m}_{t}\right\|_{\mathbf{L}^{2}\left(\Omega_{t^{\prime}}\right)}^{2}+\left\|\nabla \mathbf{u}\left(t^{\prime}\right)\right\|_{\mathbf{L}^{2}(\Omega)}^{2}+\left\|\mathbf{u}_{t}\left(t^{\prime}\right)\right\|_{\mathbf{L}^{2}(\Omega)}^{2} \leqslant C_{1},
$$

where $C_{1}$ is independent of $t$ and depends only on $|\Omega|, \mathbf{m}_{0}, \mathbf{u}_{0}$ and $\dot{\mathbf{u}}_{0}$.

\section{Preliminaries}

For time discretization, we impose a uniform partition $0=t_{0}<t_{1}<\cdots<t_{N}=T$ of the time interval $[0, T]$. The timestep size is denoted by $k=k_{j}:=t_{j+1}-t_{j}$ for $j=0, \ldots, N-1$. For each (discrete) function $\varphi$ which is continuous in time, $\varphi^{j}=\varphi\left(t_{j}\right)$ denotes the evaluation at time $t_{j}$. For the time derivatives in the conservation of momentum equation (2.10), we use difference quotients of first and second orders which are denoted by

$$
\mathrm{d}_{t} z_{i}=\frac{z_{i}-z_{i-1}}{k}, \quad \mathrm{~d}_{t}^{2} z_{i}=\frac{\mathrm{d}_{t} z_{i}-\mathrm{d}_{t} z_{i-1}}{k}=\frac{z_{i}-2 z_{i-1}+z_{i-2}}{k^{2}} .
$$

For spatial discretization, let $\mathscr{T}_{h}$ be a quasi-uniform, regular triangulation of the polyhedral bounded Lipshitz domain $\Omega \subset \mathbb{R}^{3}$ into tetrahedra. The spatial mesh size is denoted by $h$. By $\mathscr{S}^{1}\left(\mathscr{T}_{h}\right)$, we denote the lowest-order Courant finite element method (FEM) space of globally continuous and piecewise affine functions from $\Omega$ to $\mathbb{R}^{3}$, i.e.,

$$
\mathscr{S}^{1}\left(\mathscr{T}_{h}\right):=\left\{\boldsymbol{\phi}_{h} \in C\left(\bar{\Omega} ; \mathbb{R}^{3}\right):\left.\boldsymbol{\phi}_{h}\right|_{K} \in \mathscr{P}_{1}(K) \text { for all } K \in \mathscr{T}_{h}\right\}
$$

By $\mathscr{I}_{h}: C\left(\bar{\Omega} ; \mathbb{R}^{3}\right) \rightarrow \mathscr{S}^{1}\left(\mathscr{T}_{h}\right)$, we denote the nodal interpolation operator onto this space. The set of nodes of the triangulation $\mathscr{T}_{h}$ is denoted by $\mathscr{N}_{h}$.

For the discretization of the magnetization $\mathbf{m}$ in LLG (2.1a), we define the set of admissible discrete magnetizations by

$$
\mathscr{M}_{h}:=\left\{\boldsymbol{\phi}_{h} \in \mathscr{S}^{1}\left(\mathscr{T}_{h}\right):\left|\boldsymbol{\phi}_{h}(\mathbf{z})\right|=1 \text { for all } \mathbf{z} \in \mathscr{N}_{h}\right\} .
$$

Furthermore, for $\phi_{h} \in \mathscr{M}_{h}$, let

$$
\mathscr{K}_{\boldsymbol{\phi}_{h}}:=\left\{\boldsymbol{\psi}_{h} \in \mathscr{S}^{1}\left(\mathscr{T}_{h}\right): \boldsymbol{\psi}_{h}(\mathbf{z}) \cdot \boldsymbol{\phi}_{h}(\mathbf{z})=0 \text { for all } \mathbf{z} \in \mathscr{N}_{h}\right\}
$$

be the discrete tangent space associated with $\boldsymbol{\phi}_{h}$. Here, $\mathbf{x} \cdot \mathbf{y}$ stands for the usual Euclidean scalar product of $\mathbf{x}, \mathbf{y} \in \mathbb{R}^{3}$, which is sometimes also denoted by $\langle\cdot, \cdot\rangle$ to improve readability. The $L^{2}(\Omega)$ scalar product is denoted by $(\cdot, \cdot)$ throughout. Owing to the modulus constraint $\mathbf{m}_{t} \cdot \mathbf{m}=0$, and thus $|\mathbf{m}(t)|=1$ almost everywhere in $\Omega_{T}$, we discretize the time variable $\mathbf{v}\left(t_{j}\right):=\mathbf{m}_{t}\left(t_{j}\right)$ in the discrete tangent space of $\mathbf{m}_{h}^{j}$.

To discretize the equation of magnetoelasticity (2.10), we employ $\mathscr{S}_{0}^{1}\left(\mathscr{T}_{h}\right):=\mathscr{S}^{1}\left(\mathscr{T}_{h}\right) \cap H_{0}^{1}(\Omega)$. In addition, let $\mathbf{m}_{h}^{0} \in \mathscr{M}_{h}$ and $\mathbf{u}_{h}^{0}, \dot{\mathbf{u}}_{h}^{0} \in \mathscr{S}_{0}^{1}\left(\mathscr{T}_{h}\right)$ be suitable approximations of the initial data obtained, e.g., by projection. Further requirements on those initial data are specified below in Theorem 5.1. Finally, we define $\mathrm{d}_{t} \mathbf{u}_{h}^{0}$ as $\dot{\mathbf{u}}_{h}^{0}$. Throughout this work, we write $A \lesssim B$ if there holds $A \leqslant C B$ for some $h$ and $k$ independent constant $C>0$.

\section{Algorithm}

For discretization of the LLG equation, we follow the approach of Alouges (2008), which has been generalized in Alouges et al. (2011), Goldenits et al. (2011), Bruckner et al. (2012) and Goldenits 
(2012). The main idea is to introduce a new free variable $\mathbf{v} \approx \mathbf{m}_{t}$ and to interpret LLG as a linear equation in $\mathbf{v}$. This ansatz exploits the formulation

$$
\alpha \mathbf{m}_{t}+\mathbf{m} \times \mathbf{m}_{t}=\mathbf{H}_{\mathrm{eff}}-\left(\mathbf{m} \cdot \mathbf{H}_{\mathrm{eff}}\right) \mathbf{m},
$$

which is equivalent to (2.1a) under the constraint $|\mathbf{m}|=1$ almost everywhere (see, e.g., Goldenits, 2012, Lemma 1.2.1). The conservation of momentum equation is discretized in space by a standard FEM approach, and by finite differences in time. This approximation is in analogy to the one from Baňas \& Slodička (2006), where the focus is on FEM discretizations for strong solutions of (2.1) with (2.8). In addition and for computational ease, the two equations can be decoupled. We propose and analyse the following algorithm:

Algorithm 4.1 Input: Initial data $\mathbf{m}_{h}^{0}$ and $\mathbf{u}_{h}^{0}$, parameter $0 \leqslant \theta \leqslant 1, \alpha>0$. For $\ell=0, \ldots, N-1$ iterate:

(i) Compute unique solution $\mathbf{v}_{h}^{\ell} \in \mathscr{K}_{\mathbf{m}_{h}^{\ell}}$ such that for all $\boldsymbol{\varphi}_{h} \in \mathscr{K}_{\mathbf{m}_{h}^{\ell}}$, we have

$$
\alpha\left(\mathbf{v}_{h}^{\ell}, \boldsymbol{\varphi}_{h}\right)+\left(\left(\mathbf{m}_{h}^{\ell} \times \mathbf{v}_{h}^{\ell}\right), \boldsymbol{\varphi}_{h}\right)=-C_{e}\left(\nabla\left(\mathbf{m}_{h}^{\ell}+\theta k \mathbf{v}_{h}^{\ell}\right), \nabla \boldsymbol{\varphi}_{h}\right)+\left(\mathbf{h}_{\mathbf{m}}\left(\mathbf{u}_{h}^{\ell}, \mathbf{m}_{h}^{\ell}\right), \boldsymbol{\varphi}_{h}\right)-\left(\pi\left(\mathbf{m}_{h}^{\ell}\right), \boldsymbol{\varphi}_{h}\right) .
$$

(ii) Define $\mathbf{m}_{h}^{\ell+1} \in \mathscr{M}_{h}$ node-wise by $\mathbf{m}_{h}^{\ell+1}(\mathbf{z})=\left(\mathbf{m}_{h}^{\ell}(\mathbf{z})+k \mathbf{v}_{h}^{\ell}(\mathbf{z})\right) /\left|\mathbf{m}_{h}^{\ell}(\mathbf{z})+k \mathbf{v}_{h}^{\ell}(\mathbf{z})\right|$ for all $\mathbf{z} \in \mathscr{N}_{h}$.

(iii) Compute unique solution $\mathbf{u}_{h}^{\ell+1} \in \mathscr{S}_{0}^{1}\left(\mathscr{T}_{h}\right)$ such that for all $\psi_{h} \in \mathscr{S}_{0}^{1}\left(\mathscr{T}_{h}\right)$, we have

$$
\varrho\left(\mathrm{d}_{t}^{2} \mathbf{u}_{h}^{\ell+1}, \boldsymbol{\psi}_{h}\right)+\left(\lambda^{e} \boldsymbol{\varepsilon}\left(\mathbf{u}_{h}^{\ell+1}\right), \boldsymbol{\varepsilon}\left(\boldsymbol{\psi}_{h}\right)\right)=\left(\lambda^{e} \boldsymbol{\varepsilon}^{m}\left(\mathbf{m}_{h}^{\ell+1}\right), \boldsymbol{\varepsilon}\left(\boldsymbol{\psi}_{h}\right)\right) .
$$

In the above algorithm, the discrete magnetostrictive contribution is given by

$$
\left[\mathbf{h}_{\mathbf{m}}\left(\mathbf{u}_{h}^{\ell}, \mathbf{m}_{h}^{\ell}\right)\right]_{q}:=\sum_{i, j, p=1}^{3} \lambda_{i j p q}^{m} \sigma_{i j}^{h}\left(\mathbf{m}_{h}^{\ell}\right)_{p} \quad \text { with } \boldsymbol{\sigma}^{h}=\lambda^{e}\left(\boldsymbol{\varepsilon}\left(\mathbf{u}_{h}^{\ell}\right)-\boldsymbol{\varepsilon}^{m}\left(\mathbf{m}_{h}^{\ell}\right)\right) .
$$

Exploiting that we solve each of the two equations separately, we can immediately state wellposedness of Algorithm 4.1.

LEMma 4.2 Algorithm 4.1 is well defined, i.e., it admits unique discrete solutions $\left(\mathbf{v}_{h}^{\ell}, \mathbf{m}_{h}^{\ell+1}, \mathbf{u}_{h}^{\ell+1}\right)$ in each step $\ell=0, \ldots, N-1$ of the iteration. Moreover, we have $\left\|\mathbf{m}_{h}^{\ell}\right\|_{\mathbf{L}^{\infty}(\Omega)}=1$ for all $\ell=1, \ldots, N$.

Proof. We first show solvability of (4.2). We define the bilinear form:

$$
a_{1}^{\ell}(\cdot, \cdot): \mathscr{K}_{\mathbf{m}_{h}^{\ell}} \times \mathscr{K}_{\mathbf{m}_{h}^{\ell}} \rightarrow \mathbb{R}, \quad a_{1}^{\ell}(\boldsymbol{\phi}, \boldsymbol{\varphi}):=\alpha(\boldsymbol{\phi}, \boldsymbol{\varphi})+\theta C_{e} k(\nabla \boldsymbol{\phi}, \nabla \boldsymbol{\varphi})+\left(\left(\mathbf{m}_{h}^{\ell} \times \boldsymbol{\phi}\right), \boldsymbol{\varphi}\right)
$$

and the linear functional $L_{1}^{\ell}(\boldsymbol{\varphi}):=C_{e}\left(\nabla \mathbf{m}_{h}^{\ell}, \nabla \boldsymbol{\varphi}\right)+\left(\mathbf{h}_{\mathbf{m}}\left(\mathbf{u}_{h}^{\ell}, \mathbf{m}_{h}^{\ell}\right), \boldsymbol{\varphi}\right)-\left(\boldsymbol{\pi}\left(\mathbf{m}_{h}^{\ell}\right), \boldsymbol{\varphi}\right)$. Then, (4.2) is equivalent to $a_{1}^{\ell}\left(\mathbf{v}_{h}^{\ell}, \boldsymbol{\varphi}_{h}\right)=L_{1}^{\ell}\left(\boldsymbol{\varphi}_{h}\right)$ for all $\boldsymbol{\varphi}_{h} \in \mathscr{K}_{\mathbf{m}_{h}^{\ell}}$. Note that $a_{1}^{\ell}(\cdot, \cdot)$ is positive-definite for $\alpha>0$, i.e., $a^{\ell}(\boldsymbol{\varphi}, \boldsymbol{\varphi}) \geqslant \alpha\|\varphi\|_{\mathbf{L}^{2}(\Omega)}^{2}$. Thus, by exploiting finite dimension, we see that there exists a unique $\mathbf{v}_{h}^{\ell} \in \mathscr{K}_{\mathbf{m}_{h}^{\ell}}$ which solves (4.2). Owing to pointwise orthogonality of $\mathbf{m}_{h}^{\ell}$ and $\mathbf{v}_{h}^{\ell}$, and the Pythagoras theorem, we obtain $\left|\mathbf{m}_{h}^{\ell}(\mathbf{z})+k \mathbf{v}_{h}^{\ell}(\mathbf{z})\right|^{2}=\left|\mathbf{m}_{h}^{\ell}(\mathbf{z})\right|^{2}+k\left|\mathbf{v}_{h}^{\ell}(\mathbf{z})\right|^{2} \geqslant 1$ and thus even step (ii) of the above algorithm is well defined. The bound $\left\|\mathbf{m}_{h}^{\ell}\right\|_{\mathbf{L}^{\infty}(\Omega)}=1$ can be seen by the normalization at the grid points in combination with barycentric coordinates and the convexity of each tetrahedron. 
For the second equation (4.3), we consider the bilinear form:

$$
a_{2}(\cdot, \cdot): \mathscr{S}_{0}^{1}\left(\mathscr{T}_{h}\right) \times \mathscr{S}_{0}^{1}\left(\mathscr{T}_{h}\right) \rightarrow \mathbb{R}, \quad a_{2}(\zeta, \psi):=\frac{\varrho}{k^{2}}(\zeta, \psi)+\left(\lambda^{e} \boldsymbol{\varepsilon}(\zeta), \boldsymbol{\varepsilon}(\boldsymbol{\psi})\right)
$$

and the linear functional $L_{2}^{\ell}(\boldsymbol{\psi})=\left(\lambda^{e} \boldsymbol{\varepsilon}^{m}\left(\mathbf{m}_{h}^{\ell+1}\right), \boldsymbol{\varepsilon}(\boldsymbol{\psi})\right)+\frac{\varrho}{k}\left(\mathrm{~d}_{t} \mathbf{u}_{h}^{\ell}, \boldsymbol{\psi}\right)+\frac{\varrho}{k^{2}}\left(\mathbf{u}_{h}^{\ell}, \boldsymbol{\psi}\right)$, According to (2.7) and Korn's inequality (Brenner \& Scott, 2002, Theorem 11.2.16), it holds that $a_{2}(\boldsymbol{\psi}, \boldsymbol{\psi}) \gtrsim\|\boldsymbol{\psi}\|_{\mathbf{H}^{1}(\Omega)}^{2}$. With this notation, (4.3) is equivalent to $a_{2}\left(\mathbf{u}_{h}^{\ell+1}, \boldsymbol{\psi}_{h}\right)=L_{2}^{\ell}\left(\boldsymbol{\psi}_{h}\right)$ for all functions $\boldsymbol{\psi}_{h} \in \mathscr{S}_{0}^{1}\left(\mathscr{T}_{h}\right)$, and hence, admits a unique solution $\mathbf{u}_{h}^{\ell+1} \in \mathscr{S}_{0}^{1}\left(\mathscr{T}_{h}\right)$ in each step of the loop.

\section{Main Theorem}

In this section, we aim to show that the preceding algorithm indeed converges towards the correct limit. Before we start with the actual analysis, we collect some general assumptions and some more notation. Throughout, we assume that the spatial meshes $\mathscr{T}_{h}$ are uniformly shape regular and satisfy the angle condition

$$
\int_{\Omega} \nabla \zeta_{i} \cdot \nabla \zeta_{j} \leqslant 0 \quad \text { for all hat functions } \zeta_{i}, \zeta_{j} \in \mathscr{S}^{1}\left(\mathscr{T}_{h}\right) \text { with } i \neq j \text {. }
$$

This somewhat technical condition is a crucial ingredient of the convergence proof, since it yields the discrete energy decay

$$
\left\|\nabla \mathbf{m}_{h}^{\ell+1}\right\|_{\mathbf{L}^{2}(\Omega)}^{2} \leqslant\left\|\nabla\left(\mathbf{m}_{h}^{\ell}+k \mathbf{v}_{h}^{\ell}\right)\right\|_{\mathbf{L}^{2}(\Omega)}^{2} .
$$

The estimate (5.2) is a direct consequence of the inequality $\left\|\nabla \mathscr{I}_{h}(\mathbf{m} /|\mathbf{m}|)\right\|_{\mathbf{L}^{2}(\Omega)}^{2} \leqslant\|\nabla \mathbf{m}\|_{\mathbf{L}^{2}(\Omega)}^{2}$ which was proved in Bartels (2005). We like to emphasize that the angle condition is always fulfilled for tetrahedral meshes with dihedral angles that are smaller than $\pi / 2$, cf. Bartels (2005), and easily preserved for uniform mesh-refinement. For $\mathbf{x} \in \Omega$ and $t \in\left[t_{\ell}, t_{\ell+1}\right)$ and for $\gamma_{h}^{\ell} \in\left\{\mathbf{m}_{h}^{\ell}, \mathbf{v}_{h}^{\ell}, \mathbf{u}_{h}^{\ell}\right\}$, we define the time approximations

$$
\begin{aligned}
\gamma_{h k}(t, \mathbf{x}) & :=\frac{t-t_{\ell}}{k} \gamma_{h}^{\ell+1}(\mathbf{x})+\frac{t_{\ell+1}-t}{k} \gamma_{h}^{\ell}(\mathbf{x}), \\
\gamma_{h k}^{-}(t, \mathbf{x}) & :=\gamma_{h}^{\ell}(\mathbf{x}), \quad \gamma_{h k}^{+}(t, \mathbf{x}):=\gamma_{h}^{\ell+1}(\mathbf{x}) .
\end{aligned}
$$

Note that $\gamma_{h k}$ can also be written as $\gamma_{h k}(t, \mathbf{x})=\gamma_{h}^{\ell}(\mathbf{x})+\left(t-t_{\ell}\right) \mathrm{d}_{t} \gamma_{h}^{\ell+1}(\mathbf{x})$. In addition, for $t \in\left[t_{\ell}, t_{\ell+1}\right)$, we define

$$
\begin{aligned}
& \dot{\mathbf{u}}_{h k}(t, \mathbf{x}):=\mathrm{d}_{t} \mathbf{u}_{h}^{\ell}(\mathbf{x})+\left(t-t_{\ell}\right) \mathrm{d}_{t}^{2} \mathbf{u}_{h}^{\ell+1}(\mathbf{x}), \\
& \dot{\mathbf{u}}_{h k}^{-}(t, \mathbf{x}):=\mathrm{d}_{t} \mathbf{u}_{h}^{\ell}(\mathbf{x}), \quad \dot{\mathbf{u}}_{h k}^{+}(t, \mathbf{x}):=\mathrm{d}_{t} \mathbf{u}_{h}^{\ell+1}(\mathbf{x}) .
\end{aligned}
$$

The next statement is the main theorem of this work, and particularly includes the main result from Carbou et al. (2011).

THEOREM 5.1 (a) Let $\theta \in\left(\frac{1}{2}, 1\right]$ and suppose that the meshes $\mathscr{T}_{h}$ are uniformly shape regular and satisfy the angle condition (5.1). Moreover, let the general energy contribution $\pi$ be uniformly bounded in 
$\mathbf{L}^{2}\left(\Omega_{T}\right)$, i.e.,

$$
\|\boldsymbol{\pi}(\mathbf{n})\|_{\mathbf{L}^{2}\left(\Omega_{T}\right)}^{2} \leqslant C_{\boldsymbol{\pi}} \quad \text { for all }|\mathbf{n}| \in \mathbf{L}^{2}\left(\Omega_{T}\right) \text { with } \mathbf{n} \leqslant 1 \text { a.e. in } \Omega_{T},
$$

with an $\mathbf{n}$-independent constant $C_{\boldsymbol{\pi}}>0$ and assume weak convergence of the initial data, i.e., $\mathbf{m}_{h}^{0} \rightarrow$ $\mathbf{m}^{0}, \mathbf{u}_{h}^{0} \rightarrow \mathbf{u}^{0}$ in $\mathbf{H}^{1}(\Omega)$, as well as $\dot{\mathbf{u}}_{h}^{0} \rightarrow \dot{\mathbf{u}}^{0}$ in $\mathbf{L}^{2}(\Omega)$ as $h \rightarrow 0$. Under these assumptions, we have strong $\mathbf{L}^{2}\left(\Omega_{T}\right)$-convergence of $\mathbf{m}_{h k}^{-}$towards some function $\mathbf{m} \in \mathbf{H}^{1}\left(\Omega_{T}\right)$.

(b) Suppose that, in addition to the above assumptions, we have

$$
\boldsymbol{\pi}\left(\mathbf{m}_{h k}^{-}\right) \rightarrow \boldsymbol{\pi}(\mathbf{m}) \quad \text { weakly subconvergent in } \mathbf{L}^{2}\left(\Omega_{T}\right)
$$

Then, the computed FE solutions $\left(\mathbf{m}_{h k}, \mathbf{u}_{h k}\right)$ are weakly subconvergent in $\mathbf{H}^{1}\left(\Omega_{T}\right) \times \mathbf{H}^{1}\left(\Omega_{T}\right)$ towards some functions $(\mathbf{m}, \mathbf{u})$, and those weak limits $(\mathbf{m}, \mathbf{u})$ are a weak solution of LLG with magnetostriction. In particular, weak solutions exist and each weak accumulation point of $\left(\mathbf{m}_{h k}, \mathbf{u}_{h k}\right)$ is a weak solution in the sense of Definition 2.1.

The proof will be done roughly in three steps.

(i) Boundedness of the discrete quantities and energies.

(ii) Existence of weakly convergent subsequences.

(iii) Identification of the limits with weak solutions of LLG with magnetostriction.

Those three steps incorporate the same classic convergence strategy that was also employed in the seminal works (see, e.g., Bartels \& Prohl, 2006; Alouges, 2008) and for other coupled problems (see, e.g., Baňas et al., 2008, 2013) where the coupling to Maxwell's equations is considered. The approach here is, however, somewhat different, since including magnetostriction is less straightforward than, e.g., the Maxwell system.

- For the Maxwell-LLG system in Baňas et al. (2008, 2013), the coupling of LLG and Maxwell's equations is simply done via $\left(\mathbf{H}_{h}^{j}, \mathbf{v}_{h}^{j}\right)$. In contrast to this, here the coupling operators between (4.2) and (4.3) are different, and therefore the equations cannot simply be inserted into each other. Consequently, boundedness has to be shown individually for both problems (Lemma 5.3 and Proposition 5.4). The remainder is then finally estimated via Gronwall's lemma.

- The two equations are decoupled, whence the difference of two subsequent discrete solutions has to be controlled. To that end, we exploit Abel's summation in Proposition 5.4. We also refer the reader to the remark at the end of Section 5 for further details.

Altogether we apply the same ideas as in the literature, but the analytical details (especially after decoupling the two equations) are not obvious.

Next, we continue with the proof and, as mentioned, we first show the desired boundedness and start with some preliminary lemmas.

Lemma 5.2 The discrete magnetostrictive component can be estimated by the total strain of the discrete displacement, i.e.,

$$
\left\|\mathbf{h}_{\mathbf{m}}\left(\mathbf{u}_{h}^{\ell}, \mathbf{m}_{h}^{\ell}\right)\right\|_{\mathbf{L}^{2}(\Omega)}^{2} \leqslant C_{2}\left\|\boldsymbol{\varepsilon}\left(\mathbf{u}_{h}^{\ell}\right)\right\|_{\mathbf{L}^{2}(\Omega)}^{2}+C_{3},
$$

for some constants $C_{2}, C_{3}>0$ that depend only on $\bar{\lambda}$. 
Proof. By definition of the magnetostrictive part, we immediately get

$$
\left\|\mathbf{h}_{\mathbf{m}}\left(\mathbf{u}_{h}^{\ell}, \mathbf{m}_{h}^{\ell}\right)\right\|_{\mathbf{L}^{2}(\Omega)}^{2} \lesssim \bar{\lambda}\left\|\mathbf{m}_{h}^{\ell}\right\|_{\mathbf{L}^{\infty}(\Omega)}^{2}\left\|\boldsymbol{\sigma}^{h}\right\|_{\mathbf{L}^{2}(\Omega)}^{2} \lesssim\left\|\boldsymbol{\sigma}^{h}\right\|_{\mathbf{L}^{2}(\Omega)}^{2}
$$

due to the normalization step. From the definition of the discrete stress tensor and the boundedness of the material tensors, we additionally get

$$
\left\|\boldsymbol{\sigma}^{h}\right\|_{\mathbf{L}^{2}(\Omega)}^{2} \lesssim \bar{\lambda}\left\|\boldsymbol{\varepsilon}\left(\mathbf{u}_{h}^{\ell}\right)\right\|_{\mathbf{L}^{2}(\Omega)}^{2}+\left\|\boldsymbol{\varepsilon}^{m}\left(\mathbf{m}_{h}^{\ell}\right)\right\|_{\mathbf{L}^{2}(\Omega)}^{2} \lesssim\left\|\boldsymbol{\varepsilon}\left(\mathbf{u}_{h}^{\ell}\right)\right\|_{\mathbf{L}^{2}(\Omega)}^{2}+C
$$

This yields the assertion.

Lemma 5.3 For $j=1, \ldots, N$, there holds

$$
\begin{aligned}
& \left\|\nabla \mathbf{m}_{h}^{j}\right\|_{\mathbf{L}^{2}(\Omega)}^{2}+\left(\theta-\frac{1}{2}\right) k^{2} \sum_{\ell=0}^{j-1}\left\|\nabla \mathbf{v}_{h}^{\ell}\right\|_{\mathbf{L}^{2}(\Omega)}^{2}+k \sum_{\ell=0}^{j-1}\left\|\mathbf{v}_{h}^{\ell}\right\|_{\mathbf{L}^{2}(\Omega)}^{2} \\
& \leqslant C_{4}\left(\left\|\nabla \mathbf{m}_{h}^{0}\right\|_{\mathbf{L}^{2}(\Omega)}^{2}+k \sum_{\ell=0}^{j-1}\left\|\boldsymbol{\varepsilon}\left(\mathbf{u}_{h}^{\ell}\right)\right\|_{\mathbf{L}^{2}(\Omega)}^{2}+C_{5}\right)
\end{aligned}
$$

for constants $C_{4}, C_{5}>0$ that depend only on $C_{\pi}$ as well as $C_{2}$ and $C_{3}$ from the previous lemma.

Proof. In (4.2), we use the special test function $\boldsymbol{\varphi}_{h}=\mathbf{v}_{h}^{\ell} \in \mathscr{K}_{\mathbf{m}_{h}^{\ell}}$ and obtain

$$
\alpha\left(\mathbf{v}_{h}^{\ell}, \mathbf{v}_{h}^{\ell}\right)+(\underbrace{\left.\left(\mathbf{m}_{h}^{\ell} \times \mathbf{v}_{h}^{\ell}\right), \mathbf{v}_{h}^{\ell}\right)}_{=0}=-C_{e}\left(\nabla\left(\mathbf{m}_{h}^{\ell}+\theta k \mathbf{v}_{h}^{\ell}\right), \nabla \mathbf{v}_{h}^{\ell}\right)+\left(\mathbf{h}_{\mathbf{m}}\left(\mathbf{u}_{h}^{\ell}, \mathbf{m}_{h}^{\ell}\right), \mathbf{v}_{h}^{\ell}\right)-\left(\pi\left(\mathbf{m}_{h}^{\ell}\right), \mathbf{v}_{h}^{\ell}\right),
$$

whence

$$
\alpha\left\|\mathbf{v}_{h}^{\ell}\right\|_{\mathbf{L}^{2}(\Omega)}^{2}+C_{e} \theta k\left\|\nabla \mathbf{v}_{h}^{\ell}\right\|_{\mathbf{L}^{2}(\Omega)}^{2}=-C_{e}\left(\nabla \mathbf{m}_{h}^{\ell}, \nabla \mathbf{v}_{h}^{\ell}\right)+\left(\mathbf{h}_{\mathbf{m}}\left(\mathbf{u}_{h}^{\ell}, \mathbf{m}_{h}^{\ell}\right), \mathbf{v}_{h}^{\ell}\right)-\left(\boldsymbol{\pi}\left(\mathbf{m}_{h}^{\ell}\right), \mathbf{v}_{h}^{\ell}\right)
$$

Next, we use the fact that $\left\|\nabla \mathbf{m}_{h}^{\ell+1}\right\|_{\mathbf{L}^{2}(\Omega)}^{2} \leqslant\left\|\nabla\left(\mathbf{m}_{h}^{\ell}+k \mathbf{v}_{h}^{\ell}\right)\right\|_{\mathbf{L}^{2}(\Omega)}^{2}$, see (5.2), to obtain

$$
\begin{aligned}
\frac{1}{2}\left\|\nabla \mathbf{m}_{h}^{\ell+1}\right\|_{\mathbf{L}^{2}(\Omega)}^{2} \leqslant & \frac{1}{2}\left\|\nabla \mathbf{m}_{h}^{\ell}\right\|_{\mathbf{L}^{2}(\Omega)}^{2}+k\left(\nabla \mathbf{m}_{h}^{\ell}, \nabla \mathbf{v}_{h}^{\ell}\right)+\frac{k^{2}}{2}\left\|\nabla \mathbf{v}_{h}^{\ell}\right\|_{\mathbf{L}^{2}(\Omega)}^{2} \\
\leqslant & \frac{1}{2}\left\|\nabla \mathbf{m}_{h}^{\ell}\right\|_{\mathbf{L}^{2}(\Omega)}^{2}-(\theta-1 / 2) k^{2}\left\|\nabla \mathbf{v}_{h}^{\ell}\right\|_{\mathbf{L}^{2}(\Omega)}^{2} \\
& -\frac{\alpha k}{C_{e}}\left\|\mathbf{v}_{h}^{\ell}\right\|_{\mathbf{L}^{2}(\Omega)}^{2}+\frac{k}{C_{e}}\left(\mathbf{h}_{\mathbf{m}}\left(\mathbf{u}_{h}^{\ell}, \mathbf{m}_{h}^{\ell}\right), \mathbf{v}_{h}^{\ell}\right)-\frac{k}{C_{e}}\left(\pi\left(\mathbf{m}_{h}^{\ell}\right), \mathbf{v}_{h}^{\ell}\right) .
\end{aligned}
$$


We sum over the time intervals from 0 to $j-1$, which yields for any $v>0$

$$
\begin{aligned}
\frac{1}{2} \| & \nabla \mathbf{m}_{h}^{j}\left\|_{\mathbf{L}^{2}(\Omega)}^{2}+\frac{\alpha k}{C_{e}} \sum_{\ell=0}^{j-1}\right\| \mathbf{v}_{h}^{\ell} \|_{\mathbf{L}^{2}(\Omega)}^{2} \\
\leqslant & \frac{1}{2}\left\|\nabla \mathbf{m}_{h}^{0}\right\|_{\mathbf{L}^{2}(\Omega)}^{2}+\frac{k}{C_{e}} \sum_{\ell=0}^{j-1}\left[\left(\mathbf{h}_{\mathbf{m}}\left(\mathbf{u}_{h}^{\ell}, \mathbf{m}_{h}^{\ell}\right), \mathbf{v}_{h}^{\ell}\right)-\left(\boldsymbol{\pi}\left(\mathbf{m}_{h}^{\ell}\right), \mathbf{v}_{h}^{\ell}\right)\right]-(\theta-1 / 2) k^{2} \sum_{\ell=0}^{j-1}\left\|\nabla \mathbf{v}_{h}^{\ell}\right\|_{\mathbf{L}^{2}(\Omega)}^{2} \\
\leqslant & \frac{1}{2}\left\|\nabla \mathbf{m}_{h}^{0}\right\|_{\mathbf{L}^{2}(\Omega)}^{2}+\frac{k}{4 C_{e} v} \sum_{\ell=0}^{j-1}\left(\left\|\mathbf{h}_{\mathbf{m}}\left(\mathbf{u}_{h}^{\ell}, \mathbf{m}_{h}^{\ell}\right)\right\|_{\mathbf{L}^{2}(\Omega)}^{2}+\left\|\boldsymbol{\pi}\left(\mathbf{m}_{h}^{\ell}\right)\right\|_{\mathbf{L}^{2}(\Omega)}^{2}\right) \\
& +\frac{k v}{C_{e}} \sum_{\ell=0}^{j-1}\left\|\mathbf{v}_{h}^{\ell}\right\|_{\mathbf{L}^{2}(\Omega)}^{2}-(\theta-1 / 2) k^{2} \sum_{\ell=0}^{j-1}\left\|\nabla \mathbf{v}_{h}^{\ell}\right\|_{\mathbf{L}^{2}(\Omega)}^{2}=: \text { RHS. }
\end{aligned}
$$

With Lemma 5.2 and the uniform boundedness (5.3) of $\pi(\cdot)$, we further obtain

$$
\begin{aligned}
\mathrm{RHS} \lesssim & \frac{1}{2}\left\|\nabla \mathbf{m}_{h}^{0}\right\|_{\mathbf{L}^{2}(\Omega)}^{2}+\frac{k C_{2}}{4 C_{e} v} \sum_{\ell=0}^{j-1}\left(\left\|\boldsymbol{\varepsilon}\left(\mathbf{u}_{h}^{\ell}\right)\right\|_{\mathbf{L}^{2}(\Omega)}^{2}+C_{3}\right)+C_{\pi} \\
& +\frac{k v}{C_{e}} \sum_{\ell=0}^{j-1}\left\|\mathbf{v}_{h}^{\ell}\right\|_{\mathbf{L}^{2}(\Omega)}^{2}-(\theta-1 / 2) k^{2} \sum_{\ell=0}^{j-1}\left\|\nabla \mathbf{v}_{h}^{\ell}\right\|_{\mathbf{L}^{2}(\Omega)}^{2} .
\end{aligned}
$$

In total, we thus derive

$$
\begin{gathered}
\frac{1}{2}\left\|\nabla \mathbf{m}_{h}^{j}\right\|_{\mathbf{L}^{2}(\Omega)}^{2}+\frac{k}{C_{e}}(\alpha-v) \sum_{\ell=0}^{j-1}\left\|\mathbf{v}_{h}^{\ell}\right\|_{\mathbf{L}^{2}(\Omega)}^{2}+\left(\theta-\frac{1}{2}\right) k^{2} \sum_{\ell=0}^{j-1}\left\|\nabla \mathbf{v}_{h}^{\ell}\right\|_{\mathbf{L}^{2}(\Omega)}^{2} \\
\lesssim \frac{1}{2}\left\|\nabla \mathbf{m}_{h}^{0}\right\|_{\mathbf{L}^{2}(\Omega)}^{2}+\frac{k C_{2}}{4 C_{e} \nu} \sum_{\ell=0}^{j-1}\left(\left\|\boldsymbol{\varepsilon}\left(\mathbf{u}_{h}^{\ell}\right)\right\|_{\mathbf{L}^{2}(\Omega)}^{2}+C_{3}\right)+C_{\pi} .
\end{gathered}
$$

Taking $v<\alpha$ thus yields the desired result.

Given the last two lemmas, we now aim to show boundedness of the discrete quantities involved in equation (4.3), i.e., boundedness of the discrete displacement approximations. The statement of the following result is basically found in Baňas \& Slodička (2006, Lemma 3). There, however, the focus is on strong solutions of LLG and a different integrator, so that the proof and the overall analysis differ.

Proposition 5.4 For any $j=1, \ldots, N$ and $\frac{1}{2} \leqslant \theta \leqslant 1$, there holds

$$
\left\|\mathrm{d}_{t} \mathbf{u}_{h}^{j}\right\|_{\mathbf{L}^{2}(\Omega)}^{2}+\sum_{\ell=1}^{j}\left\|\mathrm{~d}_{t} \mathbf{u}_{h}^{\ell}-\mathrm{d}_{t} \mathbf{u}_{h}^{\ell-1}\right\|_{\mathbf{L}^{2}(\Omega)}^{2}+\left\|\boldsymbol{\varepsilon}\left(\mathbf{u}_{h}^{j}\right)\right\|_{\mathbf{L}^{2}(\Omega)}^{2}+\sum_{\ell=1}^{j}\left\|\boldsymbol{\varepsilon}\left(\mathbf{u}_{h}^{\ell}\right)-\boldsymbol{\varepsilon}\left(\mathbf{u}_{h}^{\ell-1}\right)\right\|_{\mathbf{L}^{2}(\Omega)}^{2} \leqslant C_{6}
$$

for some $h$ and $k$ independent constant $C_{6}>0$ which depends only on $\lambda^{\star}$ and the constants $C_{4}$ and $C_{5}$ from Lemma 5.3. 
Proof. We use $\psi_{h}=\mathbf{u}_{h}^{\ell+1}-\mathbf{u}_{h}^{\ell}$ as a test function in (4.3) and sum up for $\ell=0, \ldots, j-1$ to see

$$
\varrho\left(\mathrm{d}_{t} \mathbf{u}_{h}^{\ell+1}-\mathrm{d}_{t} \mathbf{u}_{h}^{\ell}, \mathrm{d}_{t} \mathbf{u}_{h}^{\ell+1}\right)+\left(\lambda^{e} \boldsymbol{\varepsilon}\left(\mathbf{u}_{h}^{\ell+1}\right), \boldsymbol{\varepsilon}\left(\mathbf{u}_{h}^{\ell+1}\right)-\boldsymbol{\varepsilon}\left(\mathbf{u}_{h}^{\ell}\right)\right)=\left(\lambda^{e} \boldsymbol{\varepsilon}^{m}\left(\mathbf{m}_{h}^{\ell+1}\right), \boldsymbol{\varepsilon}\left(\mathbf{u}_{h}^{\ell+1}\right)-\boldsymbol{\varepsilon}\left(\mathbf{u}_{h}^{\ell}\right)\right)
$$

Recall that the Abel summation formula shows for any $v_{\ell} \in \mathbb{R}$ and $j \geqslant 0$

$$
\sum_{\ell=0}^{j-1}\left(v_{\ell+1}-v_{\ell}, v_{\ell+1}\right)=\frac{1}{2}\left|v_{j}\right|^{2}-\frac{1}{2}\left|v_{0}\right|^{2}+\frac{1}{2} \sum_{\ell=0}^{j-1}\left|v_{\ell+1}-v_{\ell}\right|^{2}
$$

This in combination with the symmetry and the positive-definiteness (2.7) of $\lambda^{e}$ now yields

$$
\begin{aligned}
& \left\|\mathrm{d}_{t} \mathbf{u}_{h}^{j}\right\|_{\mathbf{L}^{2}(\Omega)}^{2}+\sum_{\ell=1}^{j}\left\|\mathrm{~d}_{t} \mathbf{u}_{h}^{\ell}-\mathrm{d}_{t} \mathbf{u}_{h}^{\ell-1}\right\|_{\mathbf{L}^{2}(\Omega)}^{2}+\left\|\boldsymbol{\varepsilon}\left(\mathbf{u}_{h}^{j}\right)\right\|_{\mathbf{L}^{2}(\Omega)}^{2}+\sum_{\ell=1}^{j}\left\|\boldsymbol{\varepsilon}\left(\mathbf{u}_{h}^{\ell}\right)-\boldsymbol{\varepsilon}\left(\mathbf{u}_{h}^{\ell-1}\right)\right\|_{\mathbf{L}^{2}(\Omega)}^{2} \\
& \leqslant \tilde{C}+C \sum_{\ell=1}^{j}\left(\lambda^{e} \boldsymbol{\varepsilon}^{m}\left(\mathbf{m}_{h}^{\ell}\right), \boldsymbol{\varepsilon}\left(\mathbf{u}_{h}^{\ell}\right)-\boldsymbol{\varepsilon}\left(\mathbf{u}_{h}^{\ell-1}\right)\right),
\end{aligned}
$$

for some generic constants $C, \tilde{C}>0$. Note that the terms $\left\|\mathrm{d}_{t} \mathbf{u}_{h}^{0}\right\|_{\mathbf{L}^{2}(\Omega)}^{2}=\left\|\dot{\mathbf{u}}_{h}^{0}\right\|_{\mathbf{L}^{2}(\Omega)}^{2}$ and $\left\|\boldsymbol{\varepsilon}\left(\mathbf{u}_{h}^{0}\right)\right\|_{\mathbf{L}^{2}(\Omega)}^{2}$ are uniformly bounded due to the assumed convergence of these initial data and are hidden in the constant $\tilde{C}$.

Next, we rewrite the sum on the right-hand side as

$$
\begin{aligned}
& \sum_{\ell=1}^{j}\left(\lambda^{e} \boldsymbol{\varepsilon}^{m}\left(\mathbf{m}_{h}^{\ell}\right), \boldsymbol{\varepsilon}\left(\mathbf{u}_{h}^{\ell}\right)-\boldsymbol{\varepsilon}\left(\mathbf{u}_{h}^{\ell-1}\right)\right) \\
& \quad=\left(\lambda^{e} \boldsymbol{\varepsilon}^{m}\left(\mathbf{m}_{h}^{j}\right), \boldsymbol{\varepsilon}\left(\mathbf{u}_{h}^{j}\right)\right)-\left(\lambda^{e} \boldsymbol{\varepsilon}^{m}\left(\mathbf{m}_{h}^{1}\right), \boldsymbol{\varepsilon}\left(\mathbf{u}_{h}^{0}\right)\right)-\sum_{\ell=1}^{j-1}\left(\lambda^{e} \boldsymbol{\varepsilon}^{m}\left(\mathbf{m}_{h}^{\ell+1}\right)-\lambda^{e} \boldsymbol{\varepsilon}^{m}\left(\mathbf{m}_{h}^{\ell}\right), \boldsymbol{\varepsilon}\left(\mathbf{u}_{h}^{\ell}\right)\right) \\
& \quad=\left(\lambda^{e} \boldsymbol{\varepsilon}^{m}\left(\mathbf{m}_{h}^{j}\right), \boldsymbol{\varepsilon}\left(\mathbf{u}_{h}^{j}\right)\right)-\left(\lambda^{e} \boldsymbol{\varepsilon}^{m}\left(\mathbf{m}_{h}^{1}\right), \boldsymbol{\varepsilon}\left(\mathbf{u}_{h}^{0}\right)\right)-k \sum_{\ell=1}^{j-1}\left(\lambda^{e} \mathrm{~d}_{t} \boldsymbol{\varepsilon}^{m}\left(\mathbf{m}_{h}^{\ell+1}\right), \boldsymbol{\varepsilon}\left(\mathbf{u}_{h}^{\ell}\right)\right) .
\end{aligned}
$$

For any $\eta>0$ and due to boundedness of $\lambda^{e}$, we further obtain

$$
\begin{aligned}
& \left\|\mathrm{d}_{t} \mathbf{u}_{h}^{j}\right\|_{\mathbf{L}^{2}(\Omega)}^{2}+\sum_{\ell=1}^{j}\left\|\mathrm{~d}_{t} \mathbf{u}_{h}^{\ell}-\mathrm{d}_{t} \mathbf{u}_{h}^{\ell-1}\right\|_{\mathbf{L}^{2}(\Omega)}^{2}+\left\|\boldsymbol{\varepsilon}\left(\mathbf{u}_{h}^{j}\right)\right\|_{\mathbf{L}^{2}(\Omega)}^{2}+\sum_{\ell=1}^{j}\left\|\boldsymbol{\varepsilon}\left(\mathbf{u}_{h}^{\ell}\right)-\boldsymbol{\varepsilon}\left(\mathbf{u}_{h}^{\ell-1}\right)\right\|_{\mathbf{L}^{2}(\Omega)}^{2} \\
& \lesssim 1+k \sum_{\ell=1}^{j-1}\left\|\mathrm{~d}_{t} \boldsymbol{\varepsilon}^{m}\left(\mathbf{m}_{h}^{\ell+1}\right)\right\|_{\mathbf{L}^{2}(\Omega)}^{2}+k \sum_{\ell=1}^{j-1}\left\|\boldsymbol{\varepsilon}\left(\mathbf{u}_{h}^{\ell}\right)\right\|_{\mathbf{L}^{2}(\Omega)}^{2}+\frac{1}{4 \eta}\left\|\boldsymbol{\varepsilon}^{m}\left(\mathbf{m}_{h}^{j}\right)\right\|_{\mathbf{L}^{2}(\Omega)}^{2} \\
& \quad+\eta\left\|\boldsymbol{\varepsilon}\left(\mathbf{u}_{h}^{j}\right)\right\|_{\mathbf{L}^{2}(\Omega)}^{2}+\left\|\boldsymbol{\varepsilon}^{m}\left(\mathbf{m}_{h}^{1}\right)\right\|_{\mathbf{L}^{2}(\Omega)}^{2}+\left\|\boldsymbol{\varepsilon}\left(\mathbf{u}_{h}^{0}\right)\right\|_{\mathbf{L}^{2}(\Omega)}^{2} .
\end{aligned}
$$


For sufficiently small $\eta$, this can be simplified to

$$
\begin{aligned}
& \left\|\mathrm{d}_{t} \mathbf{u}_{h}^{j}\right\|_{\mathbf{L}^{2}(\Omega)}^{2}+\sum_{\ell=1}^{j}\left\|\mathrm{~d}_{t} \mathbf{u}_{h}^{\ell}-\mathrm{d}_{t} \mathbf{u}_{h}^{\ell-1}\right\|_{\mathbf{L}^{2}(\Omega)}^{2}+\left\|\boldsymbol{\varepsilon}\left(\mathbf{u}_{h}^{j}\right)\right\|_{\mathbf{L}^{2}(\Omega)}^{2}+\sum_{\ell=1}^{j}\left\|\boldsymbol{\varepsilon}\left(\mathbf{u}_{h}^{\ell}\right)-\boldsymbol{\varepsilon}\left(\mathbf{u}_{h}^{\ell-1}\right)\right\|_{\mathbf{L}^{2}(\Omega)}^{2} \\
& \lesssim 1+k\left(\sum_{\ell=1}^{j-1}\left\|\mathrm{~d}_{t} \boldsymbol{\varepsilon}^{m}\left(\mathbf{m}_{h}^{\ell+1}\right)\right\|_{\mathbf{L}^{2}(\Omega)}^{2}+\sum_{\ell=1}^{j-1}\left\|\boldsymbol{\varepsilon}\left(\mathbf{u}_{h}^{\ell}\right)\right\|_{\mathbf{L}^{2}(\Omega)}^{2}\right) .
\end{aligned}
$$

Here, we again used convergence of the initial data. Using the boundedness and symmetry of $\lambda^{m}$ in combination with boundedness of $\left\|\mathbf{m}_{h}^{\ell}\right\|_{\mathbf{L}^{\infty}(\Omega)}$, straightforward calculation shows $\left\|\mathrm{d}_{t} \boldsymbol{\varepsilon}^{m}\left(\mathbf{m}_{h}^{\ell}\right)\right\|_{\mathbf{L}^{2}(\Omega)}^{2} \lesssim$ $\left\|\mathrm{d}_{t} \mathbf{m}_{h}^{\ell}\right\|_{\mathbf{L}^{2}(\Omega)}^{2}$, cf. Page (2013), Lemma 6.2.7. In combination with

$$
\left\|\mathrm{d}_{t} \mathbf{m}_{h}^{\ell}\right\|_{\mathbf{L}^{2}(\Omega)}^{2}=\left\|\frac{\mathbf{m}_{h}^{\ell}-\mathbf{m}_{h}^{\ell-1}}{k}\right\| \mathbf{L}^{2}(\Omega)^{2} \leqslant\left\|\mathbf{v}_{h}^{\ell-1}\right\|_{\mathbf{L}^{2}(\Omega)}^{2},
$$

cf. Alouges (2008), Alouges et al. (2011), Bruckner et al. (2012), respectively, Goldenits (2012, Lemma 3.3.2), this results in

$$
\begin{aligned}
& \left\|\mathrm{d}_{t} \mathbf{u}_{h}^{j}\right\|_{\mathbf{L}^{2}(\Omega)}^{2}+\sum_{\ell=1}^{j}\left\|\mathrm{~d}_{t} \mathbf{u}_{h}^{\ell}-\mathrm{d}_{t} \mathbf{u}_{h}^{\ell-1}\right\|_{\mathbf{L}^{2}(\Omega)}^{2}+\left\|\boldsymbol{\varepsilon}\left(\mathbf{u}_{h}^{j}\right)\right\|_{\mathbf{L}^{2}(\Omega)}^{2}+\sum_{\ell=1}^{j}\left\|\boldsymbol{\varepsilon}\left(\mathbf{u}_{h}^{\ell}\right)-\boldsymbol{\varepsilon}\left(\mathbf{u}_{h}^{\ell-1}\right)\right\|_{\mathbf{L}^{2}(\Omega)}^{2} \\
& \quad \lesssim 1+k\left(\sum_{\ell=1}^{j-1}\left\|\mathbf{v}_{h}^{\ell}\right\|_{\mathbf{L}^{2}(\Omega)}^{2}+\sum_{\ell=1}^{j-1}\left\|\boldsymbol{\varepsilon}\left(\mathbf{u}_{h}^{\ell}\right)\right\|_{\mathbf{L}^{2}(\Omega)}^{2}\right) .
\end{aligned}
$$

Next, we apply Lemma 5.3 to see

$$
\begin{aligned}
& \left\|\mathrm{d}_{t} \mathbf{u}_{h}^{j}\right\|_{\mathbf{L}^{2}(\Omega)}^{2}+\sum_{\ell=1}^{j}\left\|\mathrm{~d}_{t} \mathbf{u}_{h}^{\ell}-\mathrm{d}_{t} \mathbf{u}_{h}^{\ell-1}\right\|_{\mathbf{L}^{2}(\Omega)}^{2}+\left\|\boldsymbol{\varepsilon}\left(\mathbf{u}_{h}^{j}\right)\right\|_{\mathbf{L}^{2}(\Omega)}^{2}+\sum_{\ell=1}^{j}\left\|\boldsymbol{\varepsilon}\left(\mathbf{u}_{h}^{\ell}\right)-\boldsymbol{\varepsilon}\left(\mathbf{u}_{h}^{\ell-1}\right)\right\|_{\mathbf{L}^{2}(\Omega)}^{2} \\
& \quad \lesssim 1+\left(\left\|\nabla \mathbf{m}_{h}^{0}\right\|_{\mathbf{L}^{2}(\Omega)}^{2}+k \sum_{\ell=0}^{j-1}\left\|\boldsymbol{\varepsilon}\left(\mathbf{u}_{h}^{\ell}\right)\right\|_{\mathbf{L}^{2}(\Omega)}^{2}+k \sum_{\ell=1}^{j-1}\left\|\boldsymbol{\varepsilon}\left(\mathbf{u}_{h}^{\ell}\right)\right\|_{\mathbf{L}^{2}(\Omega)}^{2}\right) \\
& \quad \lesssim 1+k \sum_{\ell=0}^{j-1}\left\|\boldsymbol{\varepsilon}\left(\mathbf{u}_{h}^{\ell}\right)\right\|_{\mathbf{L}^{2}(\Omega)}^{2} .
\end{aligned}
$$

Application of a discrete version of Gronwall's lemma finally yields the assertion.

In order to show the desired $\mathbf{H}^{1}\left(\Omega_{T}\right)$-convergence of $\mathbf{u}$, we still need to show uniform boundedness of the $\mathbf{L}^{2}\left(\Omega_{T}\right)$-part. This result is stated in the following corollary. 
COROLlary 5.5 Owing to the boundary conditions employed, the Poincaré inequality in combination with Korn's inequality shows

$$
\begin{aligned}
\left\|\mathbf{u}_{h}^{j}\right\|_{\mathbf{L}^{2}(\Omega)}^{2}+\sum_{\ell=1}^{j}\left\|\mathbf{u}_{h}^{\ell}-\mathbf{u}_{h}^{\ell-1}\right\|_{\mathbf{L}^{2}(\Omega)}^{2} & \leqslant C_{7}\left(\left\|\nabla \mathbf{u}_{h}^{j}\right\|_{\mathbf{L}^{2}(\Omega)}^{2}+\sum_{\ell=1}^{j}\left\|\nabla\left(\mathbf{u}_{h}^{\ell}-\mathbf{u}_{h}^{\ell-1}\right)\right\|_{\mathbf{L}^{2}(\Omega)}^{2}\right) \\
& \leqslant C_{7}\left(\left\|\boldsymbol{\varepsilon}\left(\mathbf{u}_{h}^{j}\right)\right\|_{\mathbf{L}^{2}(\Omega)}^{2}+\sum_{\ell=1}^{j}\left\|\boldsymbol{\varepsilon}\left(\mathbf{u}_{h}^{\ell}-\mathbf{u}_{h}^{\ell-1}\right)\right\|_{\mathbf{L}^{2}(\Omega)}^{2}\right)
\end{aligned}
$$

for any $j=1, \ldots, N$, where the constant $C_{7}>0$ stems from Poincaré's inequality and thus only depends on the diameter of $\Omega$. According to Proposition 5.4, the right-hand side of (5.9) is uniformly bounded.

Proposition 5.4 now immediately yields boundedness of the discrete magnetizations.

COROLlary 5.6 For any $j=1, \ldots, N$, there holds

$$
\left\|\nabla \mathbf{m}_{h}^{j}\right\|_{\mathbf{L}^{2}(\Omega)}^{2}+k \sum_{\ell=0}^{j-1}\left\|\mathbf{v}_{h}^{\ell}\right\|_{\mathbf{L}^{2}(\Omega)}^{2}+\left(\theta-\frac{1}{2}\right) k^{2} \sum_{\ell=0}^{j-1}\left\|\nabla \mathbf{v}_{h}^{\ell}\right\|_{\mathbf{L}^{2}(\Omega)}^{2} \leqslant C_{8}
$$

for some constant $C_{8}>0$ which depends only on $C_{4}, C_{5}$ and $C_{6}$.

Proof. From Lemma 5.3, we obtain

$$
\begin{aligned}
& \left\|\nabla \mathbf{m}_{h}^{j}\right\|_{\mathbf{L}^{2}(\Omega)}^{2}+\left(\theta-\frac{1}{2}\right) k^{2} \sum_{\ell=0}^{j-1}\left\|\nabla \mathbf{v}_{h}^{\ell}\right\|_{\mathbf{L}^{2}(\Omega)}^{2}+k \sum_{\ell=0}^{j-1}\left\|\mathbf{v}_{h}^{\ell}\right\|_{\mathbf{L}^{2}(\Omega)}^{2} \\
& \leqslant C_{4}\left(\left\|\nabla \mathbf{m}_{h}^{0}\right\|_{\mathbf{L}^{2}(\Omega)}^{2}+k \sum_{\ell=0}^{j-1}\left\|\boldsymbol{\varepsilon}\left(\mathbf{u}_{h}^{\ell}\right)\right\|_{\mathbf{L}^{2}(\Omega)}^{2}+C_{5}\right)
\end{aligned}
$$

By utilizing Proposition 5.4, we see $k \sum_{\ell=0}^{j-1}\left\|\boldsymbol{\varepsilon}\left(\mathbf{u}_{h}^{\ell}\right)\right\|_{\mathbf{L}^{2}(\Omega)}^{2} \leqslant|T| C_{6}$. The uniform boundedness of $\left\|\nabla \mathbf{m}_{h}^{0}\right\|_{\mathbf{L}^{2}(\Omega)}$ concludes the proof.

Next, we deduce the existence of convergent subsequences.

Lemma 5.7 Let $\frac{1}{2} \leqslant \theta \leqslant 1$. Then, there exist functions $(\mathbf{m}, \mathbf{u}, \dot{\mathbf{u}}) \in \mathbf{H}^{1}\left(\Omega_{T}, \mathbb{S}^{2}\right) \times \mathbf{H}^{1}\left(\Omega_{T}\right) \times \mathbf{L}^{2}\left(\Omega_{T}\right)$ such that

$$
\begin{aligned}
& \mathbf{m}_{h k} \rightarrow \mathbf{m} \text { in } \mathbf{H}^{1}\left(\Omega_{T}\right), \\
& \mathbf{m}_{h k}, \mathbf{m}_{h k}^{ \pm} \rightarrow \mathbf{m} \text { in } L^{2}\left(\mathbf{H}^{1}\right),
\end{aligned}
$$




$$
\begin{aligned}
& \mathbf{m}_{h k}, \mathbf{m}_{h k}^{ \pm} \rightarrow \mathbf{m} \text { in } \mathbf{L}^{2}\left(\Omega_{T}\right), \\
& \mathbf{m}_{h k}, \mathbf{m}_{h k}^{ \pm} \rightarrow \mathbf{m} \text { pointwise almost everywhere in } \Omega_{T}, \\
& \mathbf{u}_{h k} \rightarrow \mathbf{u} \text { in } \mathbf{H}^{1}\left(\Omega_{T}\right), \\
& \mathbf{u}_{h k}, \mathbf{u}_{h k}^{ \pm} \rightarrow \mathbf{u} \text { in } L^{2}\left(\mathbf{H}^{1}\right), \\
& \mathbf{u}_{h k}, \mathbf{u}_{h k}^{ \pm} \rightarrow \mathbf{u} \text { in } \mathbf{L}^{2}\left(\Omega_{T}\right), \\
& \dot{\mathbf{u}}_{h k}, \dot{\mathbf{u}}_{h k}^{ \pm} \rightarrow \text { i in } \mathbf{L}^{2}\left(\Omega_{T}\right) .
\end{aligned}
$$

Here, the convergence is to be understood for a subsequence of the corresponding sequences which is successively constructed, i.e., for arbitrary spatial mesh size $h \rightarrow 0$ and timestep size $k \rightarrow 0$, there exist subindices $h_{n}$ and $k_{n}$, for which the above convergence properties are satisfied simultaneously. In addition, there exists some $\mathbf{v} \in \mathbf{L}^{2}\left(\Omega_{\tau}\right)$ with

$$
\mathbf{v}_{h k}^{-} \rightarrow \mathbf{v} \quad \text { in } \mathbf{L}^{2}\left(\Omega_{T}\right)
$$

again for the same subsequence as above.

Proof. From Proposition 5.4, Corollary 5.5 and Corollary 5.6, we immediately get boundedness of all of those sequences. A compactness argument thus allows one to successively extract convergent subsequences. Therefore, it only remains to show, that the corresponding limits coincide, i.e.,

$$
\lim \gamma_{h k}=\lim \gamma_{h k}^{-}=\lim \gamma_{h k}^{+} \quad \text { with } \gamma_{h k} \in\left\{\mathbf{m}_{h k}, \mathbf{u}_{h k}, \dot{\mathbf{u}}_{h k}\right\}
$$

To see this, we make use of the boundedness of two subsequent solutions. From Proposition 5.4, Corollary 5.5 and Corollary 5.6, we see that

$$
\begin{aligned}
& \sum_{\ell=0}^{j-1}\left\|\mathbf{m}_{h}^{\ell+1}-\mathbf{m}_{h}^{\ell}\right\|_{\mathbf{L}^{2}(\Omega)}^{2}+\sum_{\ell=0}^{j-1}\left\|\mathbf{u}_{h}^{\ell+1}-\mathbf{u}_{h}^{\ell}\right\|_{\mathbf{L}^{2}(\Omega)}^{2} \\
& \quad+\sum_{\ell=0}^{j-1}\left\|\boldsymbol{\varepsilon}\left(\mathbf{u}_{h}^{\ell+1}\right)-\boldsymbol{\varepsilon}\left(\mathbf{u}_{h}^{\ell}\right)\right\|_{\mathbf{L}^{2}(\Omega)}^{2}+\sum_{\ell=0}^{j-1}\left\|\mathrm{~d}_{t}\left(\mathbf{u}_{h}^{\ell+1}\right)-\mathrm{d}_{t}\left(\mathbf{u}_{h}^{\ell}\right)\right\|_{\mathbf{L}^{2}(\Omega)}^{2}
\end{aligned}
$$

is uniformly bounded. For the first sum, we used the inequality

$$
\left\|\mathbf{m}_{h}^{\ell+1}-\mathbf{m}_{h}^{\ell}\right\|_{\mathbf{L}^{2}(\Omega)}^{2} \leqslant k^{2}\left\|\mathbf{v}_{h}^{\ell}\right\|_{\mathbf{L}^{2}(\Omega)}^{2}
$$

(see, e.g., Alouges, 2008; Goldenits, 2012). We thus obtain

$$
\begin{aligned}
\left\|\gamma_{h k}-\gamma_{h k}^{-}\right\|_{\mathbf{L}^{2}\left(\Omega_{T}\right)}^{2} & =\sum_{\ell=0}^{N-1} \int_{t_{\ell}}^{t_{\ell+1}}\left\|\gamma_{h}^{\ell}+\frac{t-t_{\ell}}{k}\left(\gamma_{h}^{\ell+1}-\gamma_{h}^{\ell}\right)-\gamma_{h}^{\ell}\right\| \mathbf{L}^{2}(\Omega)^{2} \\
& \leqslant k \sum_{\ell=0}^{N-1}\left\|\gamma_{h}^{\ell+1}-\gamma_{h}^{\ell}\right\|_{\mathbf{L}^{2}(\Omega)}^{2} \stackrel{h \rightarrow 0}{\longrightarrow} 0,
\end{aligned}
$$


and analogously

$$
\begin{aligned}
\left\|\gamma_{h k}-\gamma_{h k}^{+}\right\|_{\mathbf{L}^{2}\left(\Omega_{T}\right)}^{2} & =\sum_{\ell=0}^{N-1} \int_{t_{\ell}}^{t_{\ell+1}}\left\|\gamma_{h}^{\ell}+\frac{t-t_{\ell}}{k}\left(\gamma_{h}^{\ell+1}-\gamma_{h}^{\ell}\right)-\gamma_{h}^{\ell+1}\right\| \mathbf{L}^{2}(\Omega)^{2} \\
& \leqslant \sum_{\ell=0}^{N-1} \int_{t_{\ell}}^{t_{\ell+1}} 2\left\|\gamma_{h}^{\ell+1}-\gamma_{h}^{\ell}\right\|_{\mathbf{L}^{2}(\Omega)}^{2} \\
& \leqslant 2 k \sum_{\ell=0}^{N-1}\left\|\gamma_{h}^{\ell+1}-\gamma_{h}^{\ell}\right\|_{\mathbf{L}^{2}(\Omega)}^{2} \stackrel{h \rightarrow 0}{\longrightarrow} 0
\end{aligned}
$$

We thus conclude that the limits $\lim \gamma_{h k}=\lim \gamma_{h k}^{ \pm}$coincide in $\mathbf{L}^{2}\left(\Omega_{T}\right)$. From the continuous inclusions $\mathbf{H}^{1}\left(\Omega_{T}\right) \Subset L^{2}\left(\mathbf{H}^{1}\right) \subseteq \mathbf{L}^{2}\left(\Omega_{T}\right)$ and the uniqueness of weak limits, we even conclude the convergence properties in $L^{2}\left(\mathbf{H}^{1}\right)$ and in $\mathbf{H}^{1}\left(\Omega_{T}\right)$, respectively. By use of the Weyl theorem, we may extract yet another subsequence to see pointwise convergence, i.e., (5.12d). From

$$
\||\mathbf{m}|-1\|_{\mathbf{L}^{2}\left(\Omega_{T}\right)} \leqslant\left\||\mathbf{m}|-\left|\mathbf{m}_{h k}^{-}\right|\right\|_{\mathbf{L}^{2}\left(\Omega_{T}\right)}+\left\|\left|\mathbf{m}_{h k}^{-}\right|-1\right\|_{\mathbf{L}^{2}\left(\Omega_{T}\right)}
$$

and

$$
\left\|\left|\mathbf{m}_{h k}^{-}(t, \cdot)\right|-1\right\|_{\mathbf{L}^{2}(\Omega)} \leqslant h \max _{t_{j}}\left\|\nabla \mathbf{m}_{h}^{\ell}\right\|_{\mathbf{L}^{2}(\Omega)} \stackrel{h \rightarrow 0}{\longrightarrow} 0,
$$

we finally conclude $|\mathbf{m}|=1$ almost everywhere in $\Omega_{T}$, which is the desired result.

In the remainder of this chapter, we will prove that the limiting tuple $(\mathbf{m}, \mathbf{u})$ is indeed a weak solution in the sense of Definition 2.1. First, we identify the limit function $\mathbf{v}$ with the time derivative of $\mathbf{m}$. The following result can be found, e.g., in Alouges (2008).

Lemma 5.8 The limit function $\mathbf{v} \in \mathbf{L}^{2}\left(\Omega_{T}\right)$ equals the time derivative of $\mathbf{m}$, i.e., $\mathbf{v}=\partial_{t} \mathbf{m}$ almost everywhere in $\Omega_{T}$.

We have now collected all ingredients for the proof of our main theorem.

Proof of Theorem 5.1. Let $(\boldsymbol{\zeta}, \boldsymbol{\psi}) \in C^{\infty}\left(\Omega_{T}\right) \times C_{c}^{\infty}\left([0, T) ; C^{\infty}(\Omega)\right)$ be arbitrary. We define test functions by $\left(\boldsymbol{\varphi}_{h}, \boldsymbol{\psi}_{h}\right)(t, \cdot):=\left(\mathscr{I}_{h}\left(\mathbf{m}_{h k}^{-} \times \zeta\right), \mathscr{I}_{h} \boldsymbol{\psi},\right)(t, \cdot)$. With the notation from above, we integrate equation (4.2) in time to obtain

$$
\begin{aligned}
\alpha \int_{0}^{T}\left(\mathbf{v}_{h k}^{-}, \varphi_{h}\right)+\int_{0}^{T}\left(\left(\mathbf{m}_{h k}^{-} \times \mathbf{v}_{h k}^{-}\right), \boldsymbol{\varphi}_{h}\right)= & -C_{e} \int_{0}^{T}\left(\nabla\left(\mathbf{m}_{h k}^{-}+\theta k \mathbf{v}_{h k}^{-}\right), \nabla \boldsymbol{\varphi}_{h}\right) \\
& +\int_{0}^{T}\left(\mathbf{h}_{\mathbf{m}}\left(\mathbf{u}_{h k}^{-}, \mathbf{m}_{h k}^{-}\right), \boldsymbol{\varphi}_{h}\right)-\int_{0}^{T}\left(\pi\left(\mathbf{m}_{h k}^{-}\right), \boldsymbol{\varphi}_{h}\right) .
\end{aligned}
$$

Then the magnetostrictive component is again given by

$$
\left[\mathbf{h}_{\mathbf{m}}\left(\mathbf{u}_{h k}^{-}, \mathbf{m}_{h k}^{-}\right)\right]_{\ell}:=\sum_{i, j, p} \lambda_{i j p q}^{m} \sigma_{i j}^{h k}\left(\mathbf{m}_{h k}^{-}\right)_{p} \quad \text { with } \boldsymbol{\sigma}^{h k}=\lambda^{e}\left(\boldsymbol{\varepsilon}\left(\mathbf{u}_{h k}^{-}\right)-\boldsymbol{\varepsilon}^{m}\left(\mathbf{m}_{h k}^{-}\right)\right) .
$$


The definition $\varphi_{h}(t, \cdot):=\mathscr{I}_{h}\left(\mathbf{m}_{h k}^{-} \times \zeta\right)(t, \cdot)$ and the approximation properties of the nodal interpolation operator show

$$
\begin{aligned}
& \int_{0}^{T}\left(\left(\alpha \mathbf{v}_{h k}^{-}+\mathbf{m}_{h k}^{-} \times \mathbf{v}_{h k}^{-}\right),\left(\mathbf{m}_{h k}^{-} \times \zeta\right)\right)+k \theta \int_{0}^{T}\left(\nabla \mathbf{v}_{h k}^{-}, \nabla\left(\mathbf{m}_{h k}^{-} \times \zeta\right)\right) \\
& \quad+C_{e} \int_{0}^{T}\left(\nabla \mathbf{m}_{h k}^{-}, \nabla\left(\mathbf{m}_{h k}^{-} \times \zeta\right)\right)-\int_{0}^{T}\left(\mathbf{h}_{\mathbf{m}}\left(\mathbf{u}_{h k}^{-}, \mathbf{m}_{h k}^{-}\right),\left(\mathbf{m}_{h k}^{-} \times \zeta\right)\right) \\
& \quad+\int_{0}^{T}\left(\pi\left(\mathbf{m}_{h k}^{-}\right),\left(\mathbf{m}_{h k}^{-} \times \zeta\right)\right)=\mathscr{O}(h) .
\end{aligned}
$$

Passing to the limit and using the strong $\mathbf{L}^{2}\left(\Omega_{T}\right)$-convergence of $\left(\mathbf{m}_{h k}^{-} \times \zeta\right)$ towards $(\mathbf{m} \times \zeta)$, we obtain

$$
\begin{gathered}
\int_{0}^{T}\left(\left(\alpha \mathbf{v}_{h k}^{-}+\mathbf{m}_{h k}^{-} \times \mathbf{v}_{h k}^{-}\right),\left(\mathbf{m}_{h k}^{-} \times \zeta\right)\right) \longrightarrow \int_{0}^{T}\left(\left(\alpha \mathbf{m}_{t}+\mathbf{m} \times \mathbf{m}_{t}\right),(\mathbf{m} \times \zeta)\right), \\
k \theta \int_{0}^{T}\left(\nabla \mathbf{v}_{h k}^{-}, \nabla\left(\mathbf{m}_{h k}^{-} \times \zeta\right)\right) \longrightarrow 0
\end{gathered}
$$

and

$$
\int_{0}^{T}\left(\nabla \mathbf{m}_{h k}^{-}, \nabla\left(\mathbf{m}_{h k}^{-} \times \zeta\right)\right) \longrightarrow \int_{0}^{T}(\nabla \mathbf{m}, \nabla(\mathbf{m} \times \zeta))
$$

as $(h, k) \rightarrow(0,0)$, cf. (Alouges, 2008, Proof of Theorem 2). Here, we have used the boundedness of $k\left\|\nabla \mathbf{v}_{h k}^{-}\right\|_{\mathbf{L}^{2}\left(\Omega_{t}\right)}^{2}$ which follows from $\theta \in\left(\frac{1}{2}, 1\right]$; see Corollary 5.6. Next, the weak convergence of $\boldsymbol{\pi}\left(\mathbf{m}_{h k}^{-}\right)$ from (5.4) yields

$$
\int_{0}^{T}\left(\boldsymbol{\pi}\left(\mathbf{m}_{h k}^{-}\right),\left(\mathbf{m}_{h k}^{-} \times \zeta\right)\right) \longrightarrow \int_{0}^{T}(\pi(\mathbf{m}),(\mathbf{m} \times \zeta)) .
$$

As for the magnetostrictive component, we have to show

$$
\mathbf{h}_{\mathbf{m}}\left(\mathbf{u}_{h k}^{-}, \mathbf{m}_{h k}^{-}\right) \rightarrow \mathbf{h}_{\mathbf{m}}(\mathbf{u}, \mathbf{m}) \quad \text { in } \mathbf{L}^{2}\left(\Omega_{T}\right),
$$

where it obviously suffices to show the desired property componentwise. With the definition from (2.6) and the boundedness of $\lambda^{m}$, a direct computation proves

$$
\left\|\boldsymbol{\varepsilon}^{m}\left(\mathbf{m}_{h k}^{-}\right)-\boldsymbol{\varepsilon}^{m}(\mathbf{m})\right\|_{\mathbf{L}^{2}\left(\Omega_{T}\right)}^{2} \lesssim\left\|\mathbf{m}_{h k}^{-}-\mathbf{m}\right\|_{\mathbf{L}^{2}\left(\Omega_{T}\right)}^{2},
$$

cf. Page (2013). The pointwise convergence of $\mathbf{m}_{h k}^{-}$from (5.12d) in combination with Lebesgue's dominated convergence theorem, now yield the strong convergence $\mathbf{m}_{h k}^{-} \cdot \zeta \rightarrow \mathbf{m} \cdot \zeta$. For any indices $i, j, p=1,2,3$ this shows

$$
\left(\left(\varepsilon^{m}\left(\mathbf{m}_{h k}^{-}\right)\right)_{i j}\left(\mathbf{m}_{h k}^{-}\right)_{p}, \zeta_{p}\right) \rightarrow\left(\varepsilon^{m}(\mathbf{m})_{i j} \mathbf{m}_{p}, \zeta_{p}\right)
$$

By definition of the magnetostrictive component, it therefore only remains to show

$$
\left(\boldsymbol{\varepsilon}\left(\mathbf{u}_{h k}^{-}\right)\right)_{i j}\left(\mathbf{m}_{h k}^{-}\right)_{p} \rightarrow \boldsymbol{\varepsilon}(\mathbf{u})_{i j} \cdot \mathbf{m}_{p} \quad \text { in } \mathbf{L}^{2}\left(\Omega_{T}\right)
$$


for any combination $i, j, p=1,2,3$ of indices. Analogous to the above, this can be seen by

$$
\left(\left(\boldsymbol{\varepsilon}\left(\mathbf{u}_{h k}^{-}\right)\right)_{i j}\left(\mathbf{m}_{h k}^{-}\right)_{p}, \zeta_{p}\right)=\left(\varepsilon\left(\mathbf{u}_{h k}^{-}\right)_{i j},\left(\mathbf{m}_{h k}^{-}\right)_{p} \zeta_{p}\right) \rightarrow\left(\boldsymbol{\varepsilon}(\mathbf{u})_{i j}, \mathbf{m}_{p} \boldsymbol{\zeta}_{p}\right)=\left(\varepsilon(\mathbf{u})_{i j} \mathbf{m}_{p}, \zeta_{p}\right)
$$

for all $\zeta \in C^{\infty}\left(\Omega_{T}\right)$, where the convergence of $\boldsymbol{\varepsilon}\left(\mathbf{u}_{h k}^{-}\right)$towards $\boldsymbol{\varepsilon}(\mathbf{u})$ particularly follows from the convergence of $\mathbf{u}_{h k}^{-}$towards $\mathbf{u}$ in $L^{2}\left(\mathbf{H}^{1}\right)$. So far, we have thus proved

$$
\begin{aligned}
\int_{0}^{T}\left(\left(\alpha \mathbf{m}_{t}+\mathbf{m} \times \mathbf{m}_{t}\right),(\mathbf{m} \times \zeta)\right)= & -C_{e} \int_{0}^{T}(\nabla \mathbf{m}, \nabla(\mathbf{m} \times \zeta)) \\
& +\int_{0}^{T}\left(\mathbf{h}_{\mathbf{m}}(\mathbf{u}, \mathbf{m}),(\mathbf{m} \times \zeta)\right)-\int_{0}^{T}(\boldsymbol{\pi}(\mathbf{m}),(\mathbf{m} \times \zeta)) .
\end{aligned}
$$

Proceeding as in Baňas et al. (2013), we conclude (2.11) by use of elementary pointwise calculations. The equality $\mathbf{m}(0, \cdot)=\mathbf{m}^{0}$ in the trace sense follows from the weak convergence $\mathbf{m}_{h k} \rightarrow \mathbf{m}$ in $\mathbf{H}^{1}\left(\Omega_{T}\right)$ and thus weak convergence of the traces. The equality $\mathbf{u}(0, \cdot)=\mathbf{u}^{0}$ follows analogously.

To prove (2.12), we argue similarly. From (4.3), we obtain

$$
\int_{0}^{T}\left(\left(\dot{\mathbf{u}}_{h k}\right)_{t}, \boldsymbol{\psi}_{h}\right)+\int_{0}^{T}\left(\lambda^{e} \boldsymbol{\varepsilon}\left(\mathbf{u}_{h k}^{+}\right), \boldsymbol{\varepsilon}\left(\boldsymbol{\psi}_{h}\right)\right)=\int_{0}^{T}\left(\lambda^{e} \boldsymbol{\varepsilon}^{m}\left(\mathbf{m}_{h k}^{+}\right), \boldsymbol{\varepsilon}\left(\boldsymbol{\psi}_{h}\right)\right)
$$

For the first summand on the left-hand side, we perform integration by parts in time and, due to the shape of $\psi_{h}$, obtain

$$
\int_{0}^{T}\left(\left(\dot{\mathbf{u}}_{h k}\right)_{t}, \boldsymbol{\psi}_{h}\right)=-\int_{0}^{T}\left(\dot{\mathbf{u}}_{h k},\left(\boldsymbol{\psi}_{h}\right)_{t}\right)+\underbrace{\left(\dot{\mathbf{u}}_{h k}(T, \cdot), \boldsymbol{\psi}_{h}(T, \cdot)\right)}=0-(\underbrace{\dot{\mathbf{u}}_{h k}(0, \cdot)}=\dot{\mathbf{u}}_{h}^{0}, \boldsymbol{\psi}_{h}(0, \cdot)) .
$$

Passing to the limit $(h, k) \rightarrow 0$, we see

$$
\int_{0}^{T}\left(\left(\dot{\mathbf{u}}_{h k}\right)_{t}, \boldsymbol{\psi}_{h}\right) \longrightarrow-\int_{0}^{T}\left(\dot{\mathbf{u}}, \boldsymbol{\psi}_{t}\right)-(\dot{\mathbf{u}}(0, \cdot), \boldsymbol{\psi}(0, \cdot))
$$

Here, we have used the assumed convergence of the initial data. From (5.12h) and the definition of $\dot{\mathbf{u}}_{h k}^{+}$, we obtain $\dot{\mathbf{u}}_{h k}^{+}=\partial_{t} \mathbf{u}_{h k}$, and therefore, by use of weak lower semicontinuity, conclude

$$
\left\|\dot{\mathbf{u}}-\partial_{t} \mathbf{u}\right\|_{\mathbf{L}^{2}(\Omega)}^{2} \leqslant \liminf \left\|\dot{\mathbf{u}}_{h k}^{+}-\partial_{t} \mathbf{u}_{h k}\right\|_{\mathbf{L}^{2}(\Omega)}^{2}=0
$$

whence $\dot{\mathbf{u}}=\partial_{t} \mathbf{u}$ almost everywhere in $\Omega_{T}$. The convergence of the terms

$$
\int_{0}^{T}\left(\lambda^{e} \boldsymbol{\varepsilon}\left(\mathbf{u}_{h k}^{+}\right), \boldsymbol{\varepsilon}\left(\boldsymbol{\psi}_{h}\right)\right) \longrightarrow \int_{0}^{T}\left(\lambda^{e} \boldsymbol{\varepsilon}(\mathbf{u}), \boldsymbol{\varepsilon}(\boldsymbol{\psi})\right)
$$

and

$$
\int_{0}^{T}\left(\lambda^{e} \boldsymbol{\varepsilon}^{m}\left(\mathbf{m}_{h k}^{+}\right), \boldsymbol{\varepsilon}\left(\boldsymbol{\psi}_{h}\right)\right) \longrightarrow \int_{0}^{T}\left(\lambda^{e} \boldsymbol{\varepsilon}^{m}(\mathbf{m}), \boldsymbol{\varepsilon}(\boldsymbol{\psi})\right)
$$

is straightforward. In summary, we have thus shown (2.12). 
It remains to show the energy estimate (2.13). From the discrete energy estimates (5.8) and (5.10), in combination with Korn's inequality, we get for any $t^{\prime} \in[0, T]$ with $t^{\prime} \in\left[t_{\ell}, t_{\ell+1}\right)$

$$
\begin{aligned}
& \left\|\nabla \mathbf{m}_{h k}^{+}\left(t^{\prime}\right)\right\|_{\mathbf{L}^{2}(\Omega)}^{2}+\left\|\mathbf{v}_{h k}^{-}\right\|_{\mathbf{L}^{2}\left(\Omega_{t^{\prime}}\right)}^{2}+\left\|\nabla \mathbf{u}_{h k}^{+}\left(t^{\prime}\right)\right\|_{\mathbf{L}^{2}(\Omega)}^{2}+\left\|\dot{\mathbf{u}}_{h k}^{+}\left(t^{\prime}\right)\right\|_{\mathbf{L}^{2}(\Omega)}^{2} \\
& \quad=\left\|\nabla \mathbf{m}_{h k}^{+}\left(t^{\prime}\right)\right\|_{\mathbf{L}^{2}(\Omega)}^{2}+\int_{0}^{t^{\prime}}\left\|\mathbf{v}_{h k}^{-}(t)\right\|_{\mathbf{L}^{2}(\Omega)}^{2}+\left\|\nabla \mathbf{u}_{h k}^{+}\left(t^{\prime}\right)\right\|_{\mathbf{L}^{2}(\Omega)}^{2}+\left\|\dot{\mathbf{u}}_{h k}^{+}\left(t^{\prime}\right)\right\|_{\mathbf{L}^{2}(\Omega)}^{2} \\
& \quad \leqslant\left\|\nabla \mathbf{m}_{h k}^{+}\left(t^{\prime}\right)\right\|_{\mathbf{L}^{2}(\Omega)}^{2}+\int_{0}^{t_{\ell+1}}\left\|\mathbf{v}_{h k}^{-}(t)\right\|_{\mathbf{L}^{2}(\Omega)}^{2}+\left\|\nabla \mathbf{u}_{h k}^{+}\left(t^{\prime}\right)\right\|_{\mathbf{L}^{2}(\Omega)}^{2}+\left\|\dot{\mathbf{u}}_{h k}^{+}\left(t^{\prime}\right)\right\|_{\mathbf{L}^{2}(\Omega)}^{2} \\
& \quad \leqslant \tilde{C}
\end{aligned}
$$

for some constant $\tilde{C}$ which is independent of $h$ and $k$. Integration in time thus yields for any measurable set $\mathfrak{T} \subseteq[0, T]$

$$
\int_{\mathfrak{T}}\left\|\nabla \mathbf{m}_{h k}^{+}\left(t^{\prime}\right)\right\|_{\mathbf{L}^{2}(\Omega)}^{2}+\int_{\mathfrak{T}}\left\|\mathbf{v}_{h k}^{-}\right\|_{\mathbf{L}^{2}\left(\Omega_{t^{\prime}}\right)}^{2}+\int_{\mathfrak{T}}\left\|\nabla \mathbf{u}_{h k}^{+}\left(t^{\prime}\right)\right\|_{\mathbf{L}^{2}(\Omega)}^{2}+\int_{\mathfrak{T}}\left\|\dot{\mathbf{u}}_{h k}^{+}\left(t^{\prime}\right)\right\|_{\mathbf{L}^{2}(\Omega)}^{2} \leqslant \int_{\mathfrak{T}} \tilde{C} .
$$

Weak semicontinuity now shows

$$
\int_{\mathfrak{T}}\|\nabla \mathbf{m}\|_{\mathbf{L}^{2}(\Omega)}^{2}+\int_{\mathfrak{T}}\left\|\mathbf{m}_{t}\right\|_{\mathbf{L}^{2}\left(\Omega_{t^{\prime}}\right)}^{2}+\int_{\mathfrak{T}}\|\nabla \mathbf{u}\|_{\mathbf{L}^{2}(\Omega)}^{2}+\int_{\mathfrak{T}}\left\|\mathbf{u}_{t}\right\|_{\mathbf{L}^{2}(\Omega)}^{2} \leqslant \int_{\mathfrak{T}} \tilde{C},
$$

which concludes the proof.

REMARK 5.9 One of the main features of the proposed algorithm is the decoupling of both equations. The analysis reveals that the main ingredient needed for the construction of a fully decoupled algorithm is a uniform bound for the sum of two subsequent solutions, i.e.,

$$
\sum_{\ell=1}^{j}\left\|\mathbf{u}_{h}^{\ell}-\mathbf{u}_{h}^{\ell+1}\right\|_{\mathbf{L}^{2}(\Omega)}^{2}+\sum_{\ell=1}^{j}\left\|\boldsymbol{\varepsilon}\left(\mathbf{u}_{h}^{\ell}-\mathbf{u}_{h}^{\ell+1}\right)\right\|_{\mathbf{L}^{2}(\Omega)}^{2}+\sum_{\ell=1}^{j}\left\|\mathbf{m}_{h}^{\ell}-\mathbf{m}_{h}^{\ell+1}\right\|_{\mathbf{L}^{2}(\Omega)}^{2} \leqslant C .
$$

On the one hand, this yields boundedness of the respective discrete solutions, as shown in Proposition 5.4 and the subsequent corollaries. On the other hand, Lemma 5.7 then yields that the limits for $\gamma_{h k}^{+}$and $\gamma_{h k}^{-}$coincide. Put explicitly, the decoupling leads to sequences of discrete solutions which are shifted in time. The above bound, however, guarantees that the limits are the same. This is why the discrete solutions of the decoupled equations converge towards a solution of the continuous coupled problem.

REMARK 5.10 Finally, we like to comment on the choice of $0 \leqslant \theta \leqslant \frac{1}{2}$ to emphasize how variants of Theorem 5.1 are read and proved.

(1) For $0 \leqslant \theta<\frac{1}{2}$, one has to bound the term $k^{2} \sum_{\ell=0}^{j-1}\left\|\nabla \mathbf{v}_{h}^{\ell}\right\|_{\mathbf{L}^{2}(\Omega)}^{2}$ in Corollary 5.6, in order to prove boundedness of the discrete quantities. This can be achieved by using an inverse estimate $\left\|\nabla \mathbf{v}_{h}^{\ell}\right\|_{\mathbf{L}^{2}(\Omega)}^{2} \lesssim\left(1 / h^{2}\right)\left\|\mathbf{v}_{h}^{\ell}\right\|_{\mathbf{L}^{2}(\Omega)}^{2}$. The latter term can then be absorbed into the term $k \sum_{\ell=0}^{j-1}\left\|\mathbf{v}_{h}^{\ell}\right\|_{\mathbf{L}^{2}(\Omega)}^{2}$ and thus yields convergence as $k / h^{2} \rightarrow 0$. 
(2) For the intermediate case $\theta=\frac{1}{2}$, the limit $k \theta \int_{0}^{T}\left(\nabla \mathbf{v}_{h k}^{-}, \nabla\left(\mathbf{m}_{h k}^{-} \times \zeta\right)\right) \rightarrow 0$ is no longer valid (see Alouges, 2008, Proof of Theorem 2). As suggested in Alouges (2008), this can be circumvented by an inverse estimate provided that $k / h \rightarrow 0$.

\section{Numerical experiments}

In this section, we provide some numerical experiments, the gain of which is twofold: First, we compare the performance of the proposed Algorithm 4.1 with the midpoint scheme from Bartels \& Prohl (2006) and Rochat (2012) for a two-dimensional blowup benchmark example. Afterwards, we revisit the blowup example and investigate the influence of the magnetostrictive component on the discrete blowup time.

\subsection{General performance}

We consider the following blowup benchmark example in two-dimensional, proposed by Bartels \& Prohl (2006): On $\Omega=(-0.5,0.5)^{2}$, we solve problem (2.9). For some fixed parameter $s \in \mathbb{R}$ and $A:=$ $(1-2|\mathbf{x}|)^{4} / s$, the initial magnetization reads

$$
\mathbf{m}_{0}(\mathbf{x})= \begin{cases}(0,0,-1) & \text { for }|\mathbf{x}| \geqslant 0.5 \\ \frac{\left(2 \mathbf{x} A, A^{2}-|\mathbf{x}|^{2}\right)}{\left(A^{2}+|\mathbf{x}|^{2}\right)} & \text { for }|\mathbf{x}| \leqslant 0.5\end{cases}
$$

This choice of $\mathbf{m}_{0}$ is motivated in Bartels et al. (2008), in order to form some singularity at the centre $\mathbf{x}=0$.

The triangulation $\mathscr{T}_{r}$ used in the numerical simulation is defined through a positive integer $r$ and consists of $2^{2 r+1}$ halved squares with edge length $h=2^{-r}$. Since this triangulation is of Delaunay type, the angle condition (5.3) is satisfied (see Alouges, 2008).

For comparison, the midpoint scheme is computed as described in Rochat (2012). The latter requires a fixed point iteration, where the tolerance is chosen as $\varepsilon=10^{-10}$, and a timestep $k_{m}>0$ that satisfies the associated mesh size condition $k_{m} \leqslant C h^{2}$ from Bartels \& Prohl (2006), Baňas et al. (2008) and Rochat (2012). In our computations for the midpoint scheme, we thus chose $k_{m}=h^{2} / 10$. The timestep size of Algorithm 4.1 is denoted by $k_{a}$, and does not depend on the spatial mesh size, i.e., as $h$ is decreased, $k_{a}$ remains fixed throughout the computations while $k_{m}$ needs to be stepwise decreased. The linear systems of Algorithm 4.1 are solved using an iterative method, and the constraint on the space $\mathscr{K}_{\mathbf{m}_{h}^{\ell}}$ is incorporated via the Lagrange multiplier approach from Goldenits et al. (2012) and Goldenits (2012).

In a first experiment, we consider the exchange-only case of LLG, and thus neglect magnetostrictive effects as well as all other field contributions, i.e., $\pi(\cdot)=0$. We compare the two algorithms for $\alpha=1, s=4, \theta=1, k_{a}=10^{-4}$ and $T=0.1[s]$. As initial value for $k_{m}$, we choose $10^{-4}$. In Table 1 , we investigate the overall computation time as well as the empirical discrete blowup time, which is the time when $|\mathbf{m}(t)|_{1, \infty}=\|\nabla \mathbf{m}(t)\|_{L^{\infty}(\Omega)}$ reaches its maximum. Note, that we did not perform the computations for $h=\frac{1}{96}$ or $h=\frac{1}{128}$ for the midpoint scheme as this would have enforced very small timesteps. We observe that Algorithm 4.1 leads to significantly lower computation time compared with the midpoint scheme. Moreover, the time of the discrete blowup shows slight variations between the different schemes. We observed that the discrete blowup times can be brought more in line with each other if the timestep size $k_{a}$ is a little decreased (not displayed). As expected, in comparison to the midpoint scheme, Algorithm 4.1 can work with larger timesteps for reasonably small $h$. 
TABLE 1 Comparison of CPU time $[s]$ and blow-up time $T_{B}$ for Algorithm 4.1 and midpoint scheme with $\alpha=1, s=4, T=0.1[s], k_{a}=10^{-4}$ and $k_{m}=\min \left\{10^{-4}, h^{2} / 10\right\}$

\begin{tabular}{|c|c|c|c|c|c|c|}
\hline \multirow[b]{2}{*}{$1 / h$} & \multicolumn{2}{|c|}{8} & \multicolumn{2}{|c|}{16} & \multicolumn{2}{|c|}{32} \\
\hline & $\mathrm{CPU}$ & $T_{B}$ & $\mathrm{CPU}$ & $T_{B}$ & $\mathrm{CPU}$ & $T_{B}$ \\
\hline $\mathrm{Alg}(4.1)$ & 20 & $>0.1$ & 79 & 0.038 & 444 & 0.024 \\
\hline \multirow[t]{3}{*}{ Mid } & 61 & $>0.1$ & 266 & 0.036 & 1510 & 0.022 \\
\hline & \multicolumn{2}{|c|}{64} & \multicolumn{2}{|c|}{96} & \multicolumn{2}{|c|}{128} \\
\hline & CPU & $T_{B}$ & $\mathrm{CPU}$ & $T_{B}$ & CPU & $T_{B}$ \\
\hline $\operatorname{Alg}(4.1)$ & 3698 & 0.037 & 16287 & 0.059 & 57078 & 0.088 \\
\hline Mid & 19209 & 0.032 & NA & NA & NA & NA \\
\hline
\end{tabular}

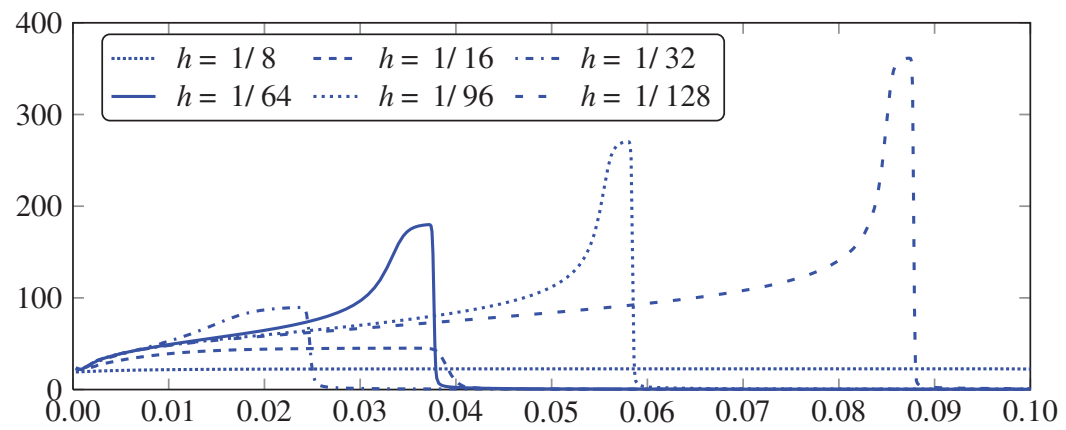

FIG. 1. $W^{1, \infty}(\Omega)$ seminorm of $\mathbf{m}_{h k}(t)$ from Algorithm 4.1 for different values of $h$, with $s=4, k_{a}=10^{-4}$.

On the other hand, the discrete blowup time seems to increase as $h$ becomes smaller, as shown in Fig. 1. This effect was not observable in Bartels \& Prohl (2006) due to the fixed-point iteration and thus the coupling of $h$ and $k_{m}$. Our empirical observation raises the question of the mere existence and behaviour of the discrete blowup in the exchange-only case. Put explicitly, the discrete blowup might be a numerical artifact stemming from insufficient spatial resolution.

In Fig. 2, we plot the discrete energy for the exchange-only case, which is defined as $E(\mathbf{m}, t)=$ $\frac{1}{2}\|\nabla \mathbf{m}(t)\|_{L^{2}(\Omega)}^{2}$, and investigate the stability of the respective algorithms when $\alpha$ becomes small. The kinks in the graph coincide with the empirical discrete blowup times. We observe that both algorithms seem to be stable, while Algorithm 4.1 provides a stronger energy decay for small values of $\alpha$.

Finally, in Fig. 3, we compare the evolution of the discrete solutions from the respective algorithms. Owing to the symmetry of the problem, we only show the evolution of the $L^{2}(\Omega)$-average ${\overline{\left\|m_{j}\right\|_{L^{2}(\Omega)}}}=$ $(1 /|\Omega|)\left(\int_{\Omega} m_{j}^{2}\right)^{1 / 2}=\left\|m_{j}\right\|_{L^{2}(\Omega)}, j=1,3$ for the $m_{1}$ and $m_{3}$ components of the magnetization, and we observe that the curves nearly coincide. Overall, we conclude that the results of our algorithm are in good agreement with those from the midpoint scheme, more feasible for small $\alpha$, throughout much faster to compute, and finally easier to implement. 


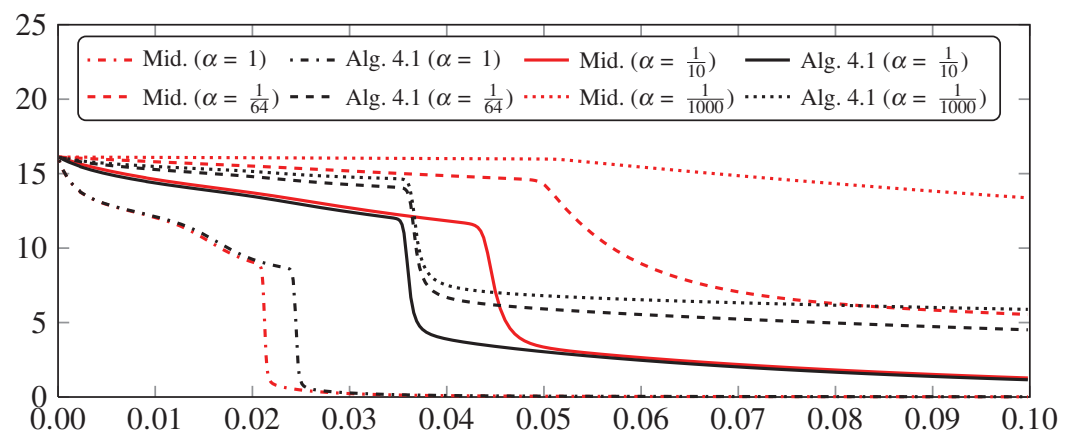

FIG. 2. Evolution of the energy for different values of $\alpha$, with $r=5, s=4, k_{a}=10^{-5}, k_{m}=10^{-5}$ and $h=\frac{1}{32}$.

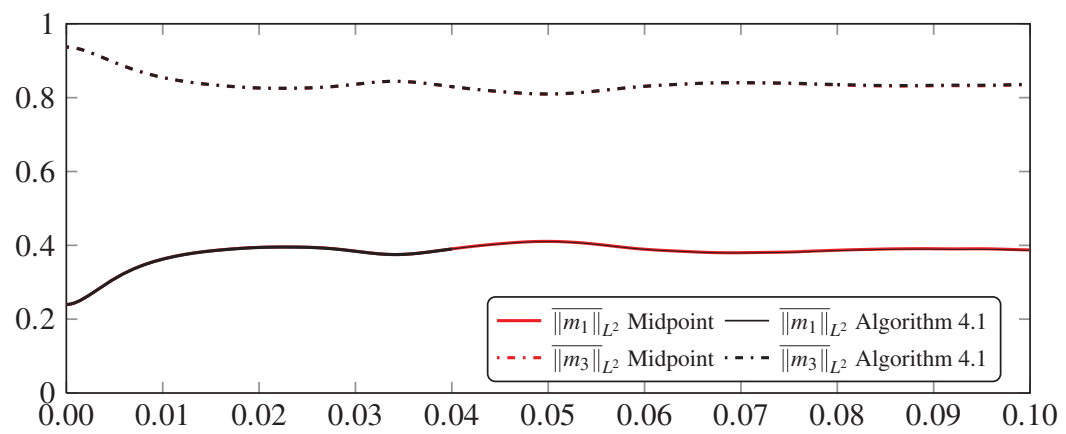

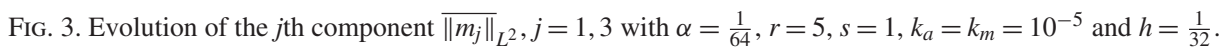

\subsection{Discrete blowup with magnetostriction}

In a second experiment, we include magnetostriction, and we take $\lambda^{e}$ and $\lambda^{m}$ to be $2 \times 2 \times 2 \times 2$ tensors with

$$
\lambda_{i j k \ell}^{e}=\left\{\begin{array}{ll}
C^{e} & i=j=k=\ell \in\{1,2\}, \\
0 & \text { else, }
\end{array} \quad \text { and } \quad \lambda_{i j k \ell}^{m}= \begin{cases}C^{m} & i=j=k=\ell \in\{1,2\}, \\
0 & \text { else, }\end{cases}\right.
$$

for given constants $C^{e}, C^{m} \geqslant 0$. In Table 2, we compare the discrete blowup times computed with Algorithm 4.1 for varying constants $C^{e}$ and $C^{m}$ and different $h>0$, where we took $\alpha=1, s=4$ and $k_{a}=10^{-4}$. For small values of $C^{e}$ and $C^{m}(\leqslant 5)$, we observe a behaviour that is very similar to the exchange-only case, i.e., the discrete blowup time increases as the mesh gets finer. As we increase the influence of the magnetostrictive component, however, we observe that the discrete blowup time stabilizes and even slightly decreases as the mesh is refined.

For further investigation, we consider the influence of $C^{e}$ and $C^{m}$ individually. In Fig. 4(left), the discrete blowup times for $\alpha=1, k_{a}=10^{-4}, h=\frac{1}{32}$ and $C^{m}=1$ for varying value of $C^{e}$ are visualized. As suggested by Table 2, the empirical blowup time decreases with increasing $C^{e}$. Figure 4(right) visualizes the behaviour of the discrete blowup time under the influence of varying parameter $C^{m}$. As before, we observe that the blowup time decreases for values of $C^{m}$, which are not too big $(\leqslant 40)$. Again, this is 
TABLE 2 Comparison of discrete blowup time for Alouges-type algorithm with $\alpha=1, s=4$, $k_{a}=10^{-4}$ and different $h$

\begin{tabular}{lcccccc}
\hline $1 / h$ & 8 & 16 & 32 & 64 & 96 & 128 \\
\hline$C^{e}=0, C^{m}=0$ & 0.106 & 0.038 & 0.024 & 0.037 & 0.059 & 0.088 \\
$C^{e}=5, C^{m}=5$ & 0.045 & 0.014 & 0.013 & 0.021 & 0.029 & 0.042 \\
$C^{e}=10, C^{m}=10$ & 0.034 & 0.012 & 0.010 & 0.011 & 0.012 & 0.014 \\
$C^{e}=40, C^{m}=10$ & - & 0.392 & 0.010 & 0.0084 & 0.0085 & 0.0090 \\
\hline
\end{tabular}
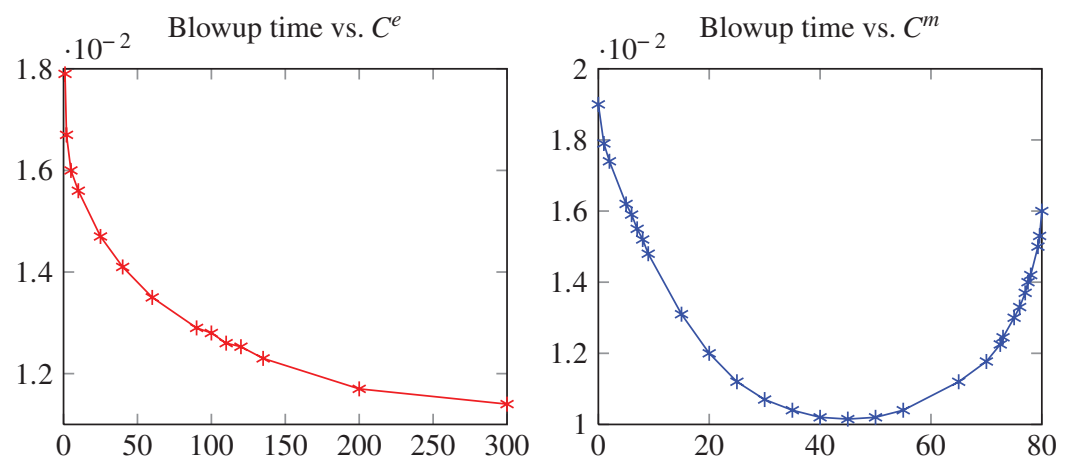

FIG. 4. Evolution of the discrete blowup time with $\alpha=1, s=4, k_{a}=10^{-4}, h=\frac{1}{32}, C^{m}=1$ for different values of $C^{m}$ (left) as well as for $C^{e}=1$ and different values of $C^{m}$ (right).

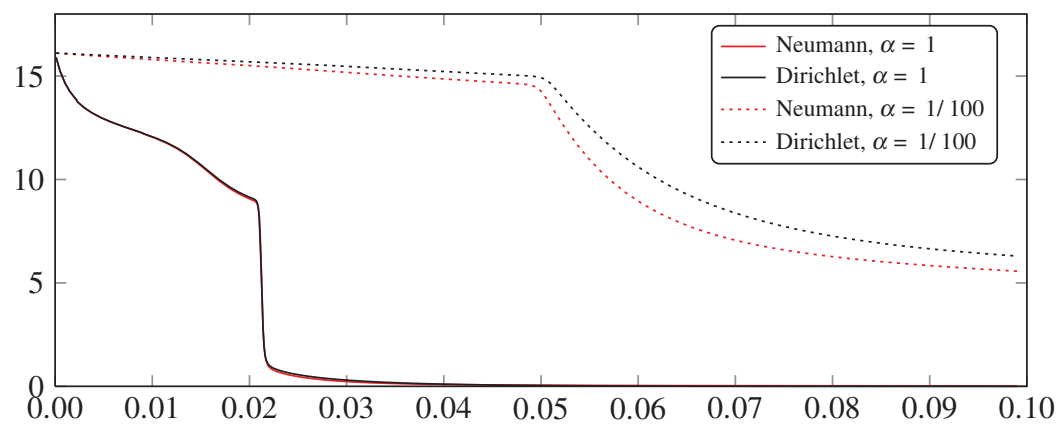

FIG. 5. Comparison of discrete blowup time between Neumann and Dirichlet boundary data.

in good agreement with the results from Table 2. After that, however, we again observe an increase in the discrete blowup time. Finally, Fig. 5 gives a comparison of the energy, and hence the times of the discrete blowup between Neumann- and Dirichlet-type boundary conditions (with $\mathbf{m}=(0,0,-1)$ at the boundary) for $h=\frac{1}{32}, k=10^{-4}, s=4$ and $\alpha \in\left\{1, \frac{1}{100}\right\}$. We observe that the type of boundary condition has very little to no influence on the time of the discrete blowup.

Altogether, we conclude that magnetostriction has a clear influence on the empirical blowup time, and that this influence is different for the two material tensors. For the exchange-only case, the empirical 
blowup time seems to be strongly mesh-dependent, and does not seem to converge towards some particular point in time. Here, it might be interesting to apply some adaptively generated meshes. Different boundary conditions have only little effect on the discrete blowup time.

\section{Funding}

M.P. and D.P. acknowledge financial support through the WWTF project MA09-029 and the FWF project P21732.

\section{REFERENCES}

Alouges, F. (2008) A new finite element scheme for Landau-Lifshitz equations. Discrete Contin. Dyn. Syst. Ser. $S, 1,187-196$.

Alouges, F., Kritsikis, E. \& Toussaint, J. (2011) A convergent finite element approximation for LandauLifshitz-Gilbert equation. Phys. B: Phys. Condens. Matter, 407, 1-5.

Alouges, F. \& Soyeur, A. (1992) On global weak solutions for Landau-Lifshitz equations: existence and nonuniqueness. Nonlinear Anal., 18, 1071-1084.

BaŇas, L., Bartels, S. \& Prohl, A. (2008) A convergent implicit finite element discretization of the MaxwellLandau-Lifshitz-Gilbert equation. SIAM J. Numer. Anal., 46, 1399-1422.

Baňas, L., Page, M. \& Praetorius, D. (2013) A convergent linear finite-element scheme for the MaxwellLandau-Lifshitz-Gilbert equation. ASC Report. Inst. Anal. Sci. Comp., Vienna University of Technology, arXiv:1303.4009.

BAŇAS, L. \& SLODIČKA, M. (2006) Error estimates for Landau-Lifshitz-Gilbert equation with magnetostriction. Appl. Numer. Math., 56, 1019-1039.

BARTELS, S. (2005) Stability and convergence of finite-element approximation schemes for harmonic maps. SIAM J. Numer. Anal., 43, 220-238.

Bartels, S., Ko, J. \& Prohl, A. (2008) Numerical analysis of an explicit approximation scheme for the LandauLifshitz-Gilbert equation. Math. Comp., 77, 773-788.

Bartels, S. \& Prohl, A. (2006) Convergence of an implicit finite element method for the Landau-Lifshitz-Gilbert equation. SIAM J. Numer. Anal., 44, 1405-1419.

Brenner, S. C. \& Scott, L. R. (2002) The Mathematical Theory of Finite Element Methods, Corr. 2nd printing. New York: Springer.

Bruckner, F., Suess, D., Feischl, M., Führer, T., Goldenits, P., Page, M. \& Praetorius, D. (2012) Multiscale modeling in micromagnetics: well-posedness and numerical integration. arXiv:1209.5548.

Carbou, G., Efendiev, M. A. \& Fabrie, P. (2011) Global weak solutions for the Landau-Lifschitz equation with magnetostriction. Math. Meth. Appl. Sci., 34, 1274-1288.

Cimrak, I. (2008) A survey on the numerics and computations for the Landau-Lifshitz equation of micromagnetism. Arch. Comput. Methods Eng., 15, 277-309.

García-Cervera, C. J. (2007) Numerical micromagnetics: a review. Bol. Soc. Esp. Mat. Apl. SeMA, 39, 103-135.

Goldenits, P. (2012) A convergent geometric time integrator to the Landau-Lifshitz-Gilbert equation (in German). Dissertation. Institute of Analysis and Scientific Computing, Vienna University of Technology.

Goldenits, P., Hrkac, G., Mayr, M., Praetorius, D. \& Suess, D. (2012) An effective integrator for the LandauLifshitz-Gilbert equation. Proc. of Mathmod 2012 Conf., 7, Part 1, 493-497.

Goldenits, P., Praetorius, D. \& Suess, D. (2011) Convergent geometric integrator for the Landau-LifshitzGilbert equation in micromagnetics. PAMM: Proc. Appl. Math. Mech., 11, 775-776.

Hubert, A. \& SchÄFer, R. (1998) Magnetic Domains. The Analysis of Magnetic Microstructures, Corr. 3rd printing. Heidelberg: Springer.

KrUŽí́, M. \& Prohl, A. (2006) Recent developments in the modeling, analysis, and numerics of ferromagnetism. SIAM Rev., 48, 439-483. 
LE, K. N. \& Tran, T. (2012) A convergent finite element approximation for the quasi-static Maxwell-LandauLifshitz-Gilbert equations. Comput. Math. Appl., 66, 1389-1402.

Page, M. (2013) On dynamical micromagnetism. Ph.D. Thesis, Institute of Analysis and Scientific Computing, Vienna University of Technology.

Prohl, A. (2001) Computational Micromagnetism. Advances in Numerical Mathematics. Stuttgart: B. G. Teubner.

Rochat, J. (2012) An implicit finite element method for the Landau-Lifshitz-Gilbert equation with exchange and magnetostriction. Master's Thesis, École Polytechnique Fédérale de Lausanne.

Visintin, A. (1985) On Landau-Lifshitz' equations for ferromagnetism. Japan J. Appl. Math., 2, 69-84. 